\title{
Automated Video Interview Personality Assessments: Reliability, Validity, and Generalizability Investigations
}

\author{
Louis Hickman ${ }^{1}$, Nigel Bosch ${ }^{2}$, Vincent $\mathrm{Ng}^{3}$, Rachel Saef ${ }^{4}$, Louis Tay ${ }^{1}$, \& Sang Eun Woo ${ }^{1}$ \\ ${ }^{1}$ Purdue University, ${ }^{2}$ University of Illinois Urbana-Champaign, ${ }^{3}$ University of Houston, \\ ${ }^{4}$ Northern Illinois University
}

\begin{abstract}
Author Note
This work was supported by a National Science Foundation Early-Concept Grant for Exploratory Research (grant number 1921111). An earlier version of this paper was presented at the Society for Industrial and Organizational Psychology 2020 Conference. Thank you to Chelsea Song and Chen Tang for their helpful comments on an earlier version of this paper. Thank you to the many members of the Well-being and Measurement Lab and the Laboratory for Understanding Careers and Individual Differences who assisted with data collection and rating the interviewees in the study.
\end{abstract}

(C) 2021, American Psychological Association. This paper is not the copy of record and may not exactly replicate the final, authoritative version of the article. Please do not copy or cite without authors' permission. The final article will be available, upon publication, via its DOI: 10.1037/apl0000695 


\begin{abstract}
Organizations are increasingly adopting automated video interviews (AVIs) to screen job applicants despite a paucity of research on their reliability, validity, and generalizability. In this study, we address this gap by developing AVIs that use verbal, paraverbal, and nonverbal behaviors extracted from video interviews to assess Big Five personality traits. We developed and validated machine learning models within (using nested cross-validation) and across three separate samples of mock video interviews (total $\mathrm{N}=1,073$ ). Also, we examined their test-retest reliability in a fourth sample $(\mathrm{N}=99)$. In general, we found that the AVI personality assessments exhibited stronger evidence of validity when they were trained on interviewer-reports rather than self-reports. When cross-validated in the other samples, AVI personality assessments trained on interviewer-reports had mixed evidence of reliability, exhibited consistent convergent and discriminant relations, used predictors that appear to be conceptually relevant to the focal traits, and predicted academic outcomes. On the other hand, there was little evidence of reliability or validity for the AVIs trained on self-reports. We discuss the implications for future work on AVIs and personality theory, and provide practical recommendations for the vendors marketing such approaches and organizations considering adopting them.
\end{abstract}

Keywords: automated video interviews, personality, machine learning, selection, validation 


\section{Automated Video Interview Personality Assessments: Reliability, Validity, and Generalizability Investigations}

Organizations are adopting automated video interviews (AVIs) that use machine learning to evaluate interviewees for early-stage applicant screening because AVIs can reduce time to hire and save organizations time and money. The use of machine learning and artificial intelligence for personnel selection can potentially provide utility beyond human-based methods and traditional assessments (e.g., Campion et al., 2016; Speer, 2018), and AVI vendors often claim that AVIs have good reliability, are free from bias, are more engaging for applicants than traditional assessments, and predict job performance (Mulfinger et al., 2020). For these reasons, AVIs have been increasing in popularity in industry. One vendor of AVIs had conducted over a million AVIs by mid-2019 (Harwell, 2019), and several other vendors are actively marketing AVI platforms (Raghavan et al., 2020). Despite their popularity, there is little psychometric evidence regarding the reliability and validity of AVIs (Chamorro-Premuzic et al., 2016; Oswald et al., 2020). This gap, together with concerns about measurement bias and fairness, has led many (including United States senators and consumer advocacy groups) to question the legality of AVIs (EPIC, 2019; Harris et al., 2019).

The present study's primary purpose is to critically evaluate the psychometric properties of automated video interview personality assessments (AVI-PAs). Specifically, we examine the following three properties: reliability (i.e., test-retest reliability; generalized coefficient of equivalence and stability); validity (i.e., convergent and discriminant relations with other variables, test content, and nomological network); and generalizability across different interview contexts. We chose to focus on personality because it predicts performance in a wide range of jobs (Barrick \& Mount, 1991; Judge \& Zapata, 2015), ample theory and research ties it to interviewee behavior (e.g., Bourdage et al., 2018; Huffcutt et al., 2011), it is commonly assessed 
by AVI vendors (e.g., HireVue; MyInterview; Yobs), and computer scientists have begun to investigate AVI-PAs (e.g., Nguyen et al., 2014; Ponce-Lopez et al., 2016). The overall process for these investigations is illustrated in Figure 1, which serves as a validation framework for developing AVI-PAs. Notably, although our study focuses on evaluating AVI-PAs, personality represents just one type of construct that can be assessed by AVIs (i.e., the method and construct are distinct; Arthur \& Villado, 2008). The framework in Figure 1 can be extended and applied to AVIs that assess other important applicant attributes—often referred to as knowledge, skills, abilities, and other characteristics (KSAOs).

To conduct our investigations, we collected four samples of mock video interviews ${ }^{1}$ (using Mechanical Turk workers and students), each comprised of different interview questions, and self- and interviewer-reported (as judged from watching the videos) Big Five personality traits. We trained machine learning models to predict interviewee self- and interviewer-reported traits in the first three samples and evaluated two sources of validity evidence (convergent and discriminant relations) using nested $k$-fold cross-validation. Next, we used those models to assess personality traits in the fourth sample to evaluate test-retest reliability and the generalized coefficient of equivalence and stability (GCES). Then we examined whether the psychometric properties (i.e., convergent and discriminant evidence of validity) of these models generalized when applied to the other samples, with each representing a unique interview context. In addition, we explored the content of the models by investigating the relative importance of verbal, paraverbal, and nonverbal behavior within the models, as well as detailing predictors common to the same-trait models. Finally, we evaluated the nomological network of AVI-PAs in relation to academic outcomes as an initial step toward investigating workplace relevant criteria.

\footnotetext{
${ }^{1}$ Many prior studies of interviews rely on student samples and mock interviews (e.g., Barrick et al., 2010; Cuddy et al., 2015; Madera \& Hebl, 2012; Swider et al., 2016; Swider et al., 2011; Van Iddekinge et al., 2005).
} 
This study makes several contributions to applied psychology. First, by examining the psychometric properties of AVI-PAs, this study addresses a practice-research gap, wherein the adoption of AVIs has outpaced research on the topic (Chamorro-Premuzic et al., 2016; Rotolo et al., 2018). In doing so, this study provides validation data to inform research and practice in this emerging area. Critically, some AVI vendors allow organizations to deploy AVIs to assess interviewees who answer interview questions that differ from those used to train the machine learning models. The present study is the first we are aware of to test whether the validity evidence of AVIs trained on one set of interview questions generalizes when tested on a new set of interview questions. Second, this study illustrates the potential value of AVIs as an alternative to self-reported personality in selection settings. Relying on self-reports in high-stake situations like personnel selection has been criticized for its susceptibility to socially desirable responding and faking (Morgeson et al., 2007; Ployhart et al., 2017; Vazire, 2010). The current investigation serves as an initial effort toward developing and validating a behavior-based personality assessment method to mitigate such concerns. Third, the paper contributes to a growing stream of research within organizational science using machine learning to automate existing assessment procedures (e.g., Campion et al., 2016; Sajjadiani et al., 2019; Speer, 2018).

\section{Automated Video Interview Personality Assessments}

Figure 1 illustrates our conceptual and operational model for investigating the construct validity of AVI-PAs. The model draws on prior theory from personality psychology (Connelly \& Ones, 2010; Funder, 1995; Vazire, 2010) and human resource management (Huffcutt et al., 2011). Our approach assumes that individuals have a true standing on the Big Five traits that is not directly observable (trait realism; Funder, 2012; Tellegen, 1991), making these traits the causal starting point of Figure 1, affecting interviewee qualifications, interviewee performance, 
self perception, and academic outcomes. Notably, Big Five personality traits represent just one example of latent constructs that AVIs could assess under this model.

Figure 1

\section{Operational model of automated video interviews}

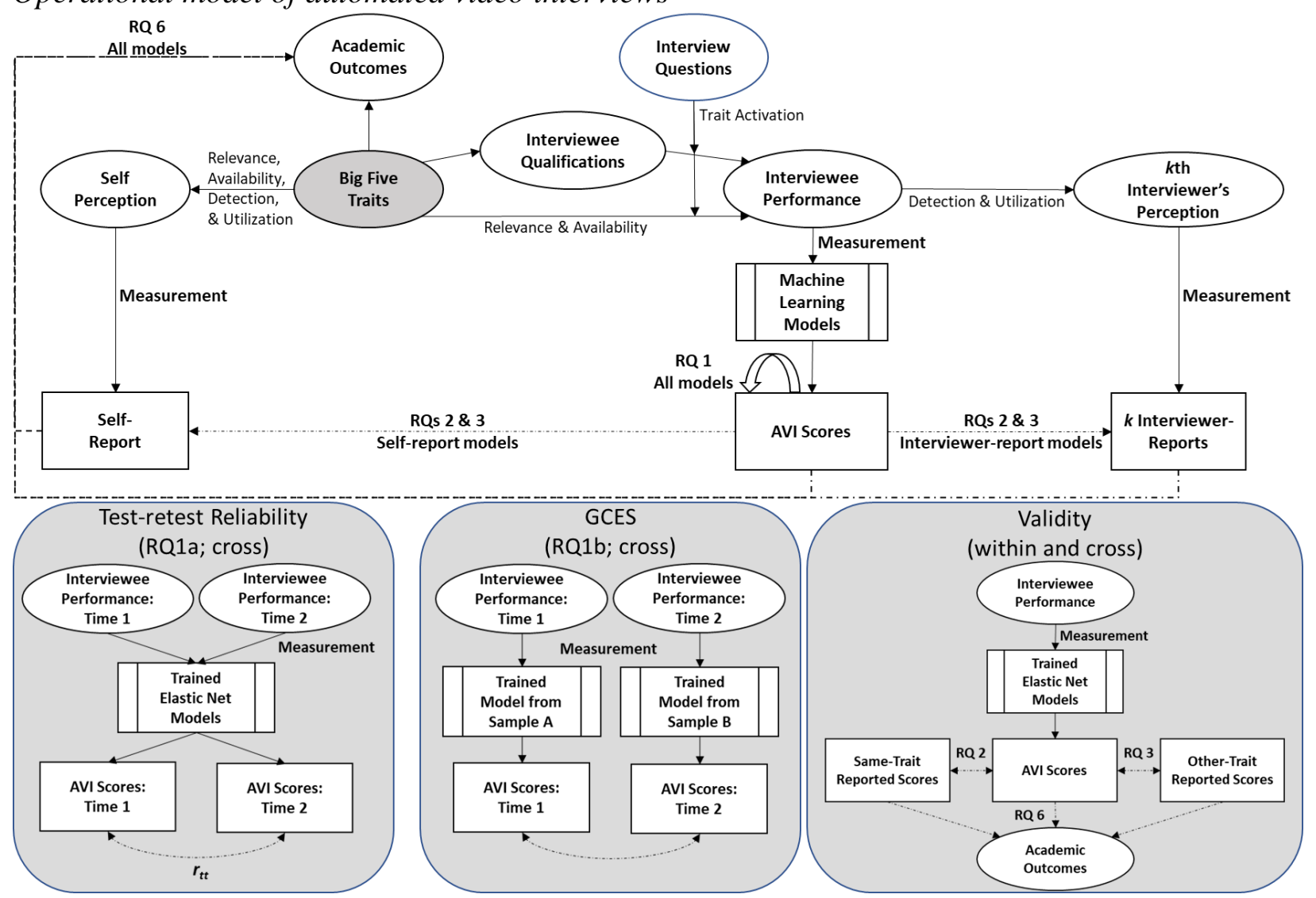

Note: $\mathrm{RQ}=$ research question. GCES $=$ generalized coefficient of equivalence and stability.

Figure is adapted from Huffcutt et al. (2011) and Connelly \& Ones (2010) for the automated video interview psychometric validation process. The lower panels provide details for how the first, second, third, and sixth Research Questions are addressed. "within" = within-sample crossvalidation; "cross" $=$ cross-sample cross-validation. $r_{t t}=$ correlations between AVI personality assessments at Time 1 and AVI personality assessments at Time 2 . GCES = generalized coefficient of equivalence and stability. For all analyses, RQs are examined separately for selfreported (with models trained on self-reports) and interviewer-reported (with models trained on interviewer-reports) traits.

Following Connelly and Ones (2010), the model in Figure 1 recognizes that neither selfnor interviewer-reports are direct representations of latent traits. The Realistic Accuracy Model (RAM; Funder, 1995) posits that only certain behavioral cues can be considered expressions of a 
given trait (i.e., relevance). Further, only some of the relevant behavioral cues are directly observable, and such cues differ in the quantity of their expression (i.e., availability). This process affects both self perception and the interviewer's perception (Connelly \& Ones, 2010) because internal behaviors relating to thoughts and feelings are not readily available to observers, while many outward behaviors, such as facial expressions, are not available to the self (per the self-other knowledge asymmetry model of personality judgment; SOKA; Vazire, 2010). Therefore, self-reports may provide more accurate information than observer-reports for less visible, internal traits (e.g., openness, emotional stability). In contrast, the reverse may be true for highly visible traits (e.g., extraversion) for which many behavioral cues are available.

Within employment interviews, personality traits affect interviewee performance (i.e., the interviewee's in situ behavior) both directly and indirectly through their effects on acquiring jobrelevant interviewee qualifications, including declarative knowledge, procedural knowledge, and motivation (Huffcutt et al., 2011). Different interview questions represent different situational features - for example, the use of situational or past behavioral questions affects the type of impression management interviewees tend to use, regardless of their personality (Peeters \& Lievens, 2006). Interview questions may differ in how trait-relevant they are, which can cause differences in the relevance and availability of behaviors elicited by a given set of interview questions (e.g., Tett \& Burnett, 2003). In other words, interviewee performance is a function of both the interviewee's individual differences and interview design (i.e., the person and the situation; Mischel \& Shoda, 1995; Huffcutt et al., 2011). Therefore, AVI-PAs trained on one set of interview questions may not generalize to a new set of interview questions to the extent that the interview questions in the training data affect the relationship between personality and interviewee performance. 
In addition to differences in behavior relevance and availability between interview questions, raters differ in their ability to notice trait-relevant behavioral cues (detection) and to combine the cues appropriately to form trait impressions (utilization; Funder, 1995). Again, as illustrated in Figure 1, this process affects both self perception and the interviewer's perception (Connelly \& Ones, 2010). The limitations of self-reports are well-known-even outside of employment contexts where faking is a concern (e.g., Morgeson et al., 2007). People are motivated by self-serving biases to protect and enhance their self-view, which can bias selfreports (although not always via simple score elevation; Vazire, 2010). In other words, both selfand interviewer-reports are affected by the rater's ability to utilize cues ${ }^{2}$.

Further, both the self and observers must then take these trait impressions and convert them into measurement scores (i.e., self-report; $k$ interviewer-reports). Self-report involves one's identity and self-serving biases, whereas interviewer-reports are based on the reputation (Hogan, 1991) gleaned from observing interviewee performance in addition to any potential perceptual biases. Both operationalizations involve some degree of measurement error and different types of biases (Connelly \& Ones, 2010; Huffcutt et al., 2011). Simply, both self- and interviewer-reports are imperfect sources of trait information, each with distinct strengths and weaknesses that may affect the validity of AVI-PAs trained on them.

\footnotetext{
${ }^{2}$ Self-serving biases are particularly likely to affect the proper utilization of cues when self-reporting traits for which social value is attached to one's trait standing, known as evaluative traits. Therefore, self-reports of personality may provide more accurate information compared to observer-reports for non-evaluative traits (e.g., extraversion, emotional stability), whereas the reverse may be true for highly evaluative traits (e.g., agreeableness, conscientiousness, and the intellect facet of openness; e.g., John \& Robins, 1993).

Research on the good judge vis-à-vis observers has uncovered several, albeit somewhat inconsistent, findings regarding who tends to be better at detecting and utilizing cues for trait perception. For example, observers tend to provide more accurate personality judgments when they have higher general mental ability and, more specifically, dispositional intelligence (i.e., knowledge about how personality traits relate to behavior; Christiansen et al., 2005; De Kock et al., 2015; Powell \& Bourdage, 2016). The rationale is that higher levels of ability improve one's capacity for "perceiving relations between past and present activities, between expressive behavior and inner traits, between cause and effect" (Allport, 1937, p. 514).
} 
Automated video interviews use computers to quantify interviewees' verbal, paraverbal (e.g., speech rate, voice quality) and nonverbal (e.g., smiles, visual attention) behaviors. Table 1 summarizes the interviewee behaviors that are operationalized in the present study. These verbal, paraverbal, and nonverbal behaviors are then used as predictors in machine learning models, a form of empirical keying, to assess interviewee (i.e., AVI scores) personality traits and other important KSAOs (e.g., Naim et al., 2018; Nguyen et al., 2014). Because AVI scores and interviewer-reports use interviewee performance as direct inputs for person perception, AVI-PAs are likely to model interviewer-reports more successfully.

For AVI-PAs to successfully model either self- or interviewer-reports, the focal KSAOs must be reflected in interviewee performance, which consists of verbal, paraverbal, and nonverbal behaviors (Huffcutt et al., 2011). Our assumption here is that personality (directly and indirectly) affects interviewee performance, and computers can capture useful information about personality in a video interview by extracting these three types of behavior (see Figure 1). In line with this, research has shown that interviewees with high self-reported conscientiousness tend to engage in more honest and less deceptive self-promotion impression management (i.e., verbal behavior; Bourdage et al., 2018). Interviewees with high self-reported emotional stability speak faster and pause less (i.e., paraverbal behavior; Feiler \& Powell, 2016). Further, interviewees with high self-reported agreeableness display more eye contact, open postures, and friendly facial expressions (i.e., nonverbal behavior; Kristof-Brown et al., 2002). These relationships are important, as interviewee performance (i.e., verbal, paraverbal, and nonverbal behavior) influences interviewer judgments of interviewee personality, judgments that are commonly made in personnel selection (Cortina et al., 2000; Huffcutt et al., 2001). For instance, certain paraverbal behaviors (i.e., pitch variability, higher speech rate, amplitude variability; DeGroot \& 
Gooty, 2009) influence interviewer judgments of extraversion, emotional stability, and openness.

Further, nonverbal behavior (e.g., open posture, eye contact) influences interviewer judgments of all Big Five interviewee traits (DeGroot \& Gooty, 2009).

Table 1

Descriptions and operationalizations of three types of interviewee behavior in present study

\begin{tabular}{|c|c|c|c|}
\hline Behavior & Definition & Operationalization & $\begin{array}{l}\text { Tools for } \\
\text { Extracting }\end{array}$ \\
\hline Verbal & $\begin{array}{l}\text { What interviewees say; } \\
\text { the content of their } \\
\text { response }\end{array}$ & $\begin{array}{l}\text { - Word count } \\
\text { - Proportion of words longer than } \\
\text { six letters } \\
\text { - } 70 \text { LIWC dictionaries } \\
\text { - } n \text {-grams with } n=1,2 \text { (i.e., } \\
\text { words and two-word phrases) }\end{array}$ & $\begin{array}{l}\text { - IBM Watson } \\
\text { Speech to Text } \\
\text { - LIWC } \\
\text { - tm R package }\end{array}$ \\
\hline Paraverbal & $\begin{array}{l}\text { How interviewees sound } \\
\text { when delivering their } \\
\text { responses }\end{array}$ & $\begin{array}{l}\text { - Pitch } \\
\text { - Jitter } \\
\text { - Frequency } \\
\text { - Shimmer } \\
\text { - Loudness } \\
\text { - Harmonics-to-Noise ratio } \\
\text { - Alpha ratio } \\
\text { - Hammarberg index } \\
\text { - Spectral slope } \\
\text { - Loudness peaks per second } \\
\text { - Length of continuously voiced } \\
\text { regions } \\
\text { - Length of continuously } \\
\text { unvoiced regions } \\
\text { - Voiced segments per second }\end{array}$ & - openSMILE \\
\hline Nonverbal & $\begin{array}{l}\text { What interviewees do } \\
\text { (e.g., facial expressions, } \\
\text { posture) }\end{array}$ & $\begin{array}{l}\text { - Head pose } \\
\text { - } 19 \text { facial action units activation } \\
\text { intensity } \\
\text { - Mean } \\
\text { - Standard deviation } \\
\text { - Kurtosis } \\
\text { - Skewness } \\
\text { - Facial action unit } \\
\text { cooccurrences }\end{array}$ & - OpenFace \\
\hline
\end{tabular}

In terms of applying AVIs to assess Big Five personality traits, prior studies in computer 
science found that AVI-PAs can converge strongly with interviewer-reported traits when crossvalidated on holdout data drawn from the same sample (see Table 2 for a review of AVI-PA studies; Biel et al., 2013; Chen et al., 2016, 2018; Nguyen et al., 2014; Nguyen \& Gatica-Perez, 2016; Ponce-López et al., 2016). For example, Chen et al. (2018) found that their machine learning models accurately classified interviewees as having high or low standing on interviewer-reported traits (macro F-1 score, or the harmonic mean of precision and recall, was approximately .80 for each trait). However, despite this promising initial evidence, critical psychometric issues remain in terms of reliability, validity, and generalizability that still need to be examined (Hickman, Saef, et al., 2020).

Table 2

Review of prior studies of AVI-PAs (all trained and tested on interviewer-reports)

\begin{tabular}{|c|c|c|c|c|}
\hline Study & $\mathrm{N}$ & $\begin{array}{l}\text { Interview } \\
\text { characteristics }\end{array}$ & $\begin{array}{l}\text { Cross-validation } \\
\text { strategy }\end{array}$ & Reported accuracy \\
\hline $\begin{array}{l}\text { Biel et al. } \\
\text { (2013) }\end{array}$ & 408 & $\begin{array}{l}\text { Video blogs from } \\
\text { YouTube }\end{array}$ & 10-fold & $\begin{array}{l}\mathrm{E}=.48 ; \mathrm{A}=.39 ; \mathrm{C}=.22 \\
\mathrm{ES}=.23 ; \mathrm{O}=.17\left(\mathrm{R}^{2}\right)\end{array}$ \\
\hline $\begin{array}{l}\text { Chen et al. } \\
\text { (2016) }\end{array}$ & 36 & $\begin{array}{l}\text { Video interview; } 12 \\
\text { PBQs }\end{array}$ & Leave-one-out & $\begin{array}{l}\mathrm{E}=.44 ; \mathrm{A}=.38 ; \mathrm{C}=.34 \\
\mathrm{ES}=.40 ; \mathrm{O}=.35(r)\end{array}$ \\
\hline $\begin{array}{l}\text { Chen et al. } \\
\text { (2018) }\end{array}$ & 260 & $\begin{array}{l}\text { Video interview; } 8 \\
\text { PBQs }\end{array}$ & $\begin{array}{l}20 \% \text { holdout test } \\
\text { sample }\end{array}$ & $\begin{array}{l}\mathrm{E}=.78 ; \mathrm{A}=.84 ; \mathrm{C}=.86 ; \\
\mathrm{ES}=.83 ; \mathrm{O}=.81(\mathrm{~F}-1 \\
\text { score; used median split } \\
\text { to classify high/low })\end{array}$ \\
\hline $\begin{array}{l}\text { Nguyen \& } \\
\text { Gatica-Perez } \\
\text { (2016) }\end{array}$ & 939 & $\begin{array}{l}\text { Video resumes from } \\
\text { YouTube (123.5 s } \\
\text { median length) }\end{array}$ & 10 -fold & $\begin{array}{l}\mathrm{E}=.27 ; \mathrm{A}=.06 ; \mathrm{C}=.03 ; \\
\mathrm{ES}=.00 ; \mathrm{O}=.20\left(\mathrm{R}^{2}\right)\end{array}$ \\
\hline $\begin{array}{l}\text { Nguyen et al. } \\
\text { (2014) }\end{array}$ & 62 & $\begin{array}{l}\text { Face-to-face; } 4 \\
\text { unstructured questions } \\
\& 4 \text { PBQs }\end{array}$ & Leave-one-out & $\mathrm{C}=.04 ; \mathrm{ES}=.27\left(\mathrm{R}^{2}\right)$ \\
\hline $\begin{array}{l}\text { Ponce-Lopez } \\
\text { et al. (2016) }\end{array}$ & 10,000 & $\begin{array}{l}15 \text { second clips from } \\
\text { YouTube videos }\end{array}$ & $\begin{array}{l}20 \% \text { holdout test } \\
\text { sample }\end{array}$ & $\begin{array}{l}\mathrm{E}=.52 ; \mathrm{A}=.34 ; \mathrm{C}=.54 \\
\mathrm{ES}=.47 ; \mathrm{O}=.44\left(\mathrm{R}^{2}\right)\end{array}$ \\
\hline
\end{tabular}

Note $\mathrm{PBQ}=$ past behavioral question. $\mathrm{E}=$ extraversion. $\mathrm{A}=$ agreeableness. $\mathrm{C}=$ conscientiousness. $\mathrm{ES}=$ emotional stability. $\mathrm{O}=$ openness. In some of these studies, $\mathrm{R}^{2}$ is not directly translatable to $r$ because the formula they used to calculate the coefficient of determination allows it to take on negative values. 


\section{Reliability}

Reliability is an essential piece of psychometric evidence for any assessment, as assessments must be reliable to be valid (Lord \& Novick, 1968; AERA et al., 2014). Yet, to our knowledge, there is no evidence to suggest that scores generated from AVIs are reliable. This is also a shortcoming of most research using machine learning and digital footprints (e.g., Facebook likes) to assess personality traits (Bleidorn \& Hopwood, 2019; Tay et al., 2020), which still would not address the reliability of AVIs.

\section{Test-retest Reliability}

The machine learning algorithms used to develop AVIs engage in a form of empirical keying by selecting and weighting behavioral cues (i.e., verbal, paraverbal, and nonverbal behaviors) to maximize convergence with human reported personality assessments. Researchers generally use Cronbach's alpha to index scale reliability, but this may not be appropriate for empirically keyed scales. Empirically keyed scales tend to select heterogeneous items that a) maximize overall correlation with criteria and thus lead to b) low interitem correlations, resulting in scales that tend not to be internally consistent (Simms, 2008).

Test-retest reliability (i.e., coefficient of stability) is used to examine construct-irrelevant variance specific to occasions (see the lower-left panel of Figure 1). Occasions represent a source of construct-irrelevant variance that may contaminate measures (i.e., transient error). Test-retest reliability is fundamental to testing because the central concern of reliability is whether a person's scores would converge if tested more than once (Cronbach, 1990). Indeed, test-retest reliability is more predictive of personality scale validity than internal consistency reliability (McCrae et al., 2011). Further, test-retest reliability is considered the ideal index of empirically keyed scales' reliability (Cucina et al., 2019). Consequently, test-retest reliability is arguably 
more appropriate than internal consistency for estimating AVI-PA reliability.

However, interview test-retest reliability is often low since interviewees may practice and improve, have fluctuations in anxiety and mood, or (as noted by a reviewer) simply talk about different events than the prior interview because constructed response format assessments allow for a wider range of responses than multiple-choice tests or Likert-type scales. Further, interviewers may be replaced with new ones. For instance, Schleicher et al. (2010) found that behavioral, situational, and experience/interest interviews conducted one year apart had testretest reliabilities of $r=.30, .35$, and .26 , respectively $(\mathrm{N}=2,060)$. These values would generally be unacceptable for other types of assessments. Unfortunately, considering the paucity of research on the subject, it is unclear what makes for adequate test-retest reliability in employment interviews (Huffcutt et al., 2013). On the other hand, personality scales tend to exhibit relatively high test-retest reliability. Costa and McCrae (1991) reported 6-year test-retest reliabilities ranging from a low of $r=.63$ for agreeableness to a high of $r=.83$ for emotional stability and openness. High test-retest reliability increases confidence that a measure captures true score variance across occasions.

\section{Generalized Coefficient of Equivalence and Stability (GCES)}

While test-retest reliability is useful, it is also important to ascertain the extent that AVI scores are stable over time when using different AVI-PA models. In other words, we are conceptually interested in the generalizability of the test-retest reliability, not merely in a single AVI-PA but across alternate forms (i.e., similar measures of the same construct). In this case, AVI-PA models trained on different data that use the same set of behaviors as potential predictors can be considered alternate forms. The GCES provides such an estimate (see the lower-center panel of Figure 1) by correlating the scores of an AVI-PA at one time point with the 
scores of another AVI-PA at a second time point. In doing so, the GCES calibrates multiple sources of error and estimates the proportion of construct variance to observed variance (Le et al., 2009) ${ }^{3}$. We adopt the GCES and use it alongside test-retest reliability to provide initial evidence regarding the reliability of AVI-PAs.

Research Question 1: How reliable are AVI-PAs, in terms of (a) test-retest reliability and

(b) the generalized coefficient of equivalence and stability?

Notably, high reliability estimates can be observed in the absence of construct validity due to method variance and other causes of inflated intercorrelations. Due to this limitation, it is critical to go beyond reliability and investigate multiple sources of validity evidence.

\section{Validity}

Validity evidence regarding an assessment procedure should show that scores derived from the procedure adequately represent the constructs of interest. Such evidence provides the basis for interpreting score meaning and using the procedure for decision-making (e.g., personnel selection; Messick, 1989). Therefore, in the current context, we are not so much interested in verbal, paraverbal, and nonverbal behaviors per se, but rather how indicative the scores produced by models trained to use these behaviors are of Big Five personality traits and, thus, justifiable for use in personnel selection. Following the Standards for Educational and Psychological Testing (in short, the Standards; AERA et al., 2014) and the Principles for the Validation and Use of Personnel Selection Procedure (SIOP, 2018), we examine several sources of AVI-PA validity evidence in this study, including convergent and discriminant relations, machine learning model content, and nomological network in relation to academic outcomes (as shown in the lower-right panel of Figure 1). Under the unitarian conception of validity, this study's

\footnotetext{
${ }^{3}$ Although the GCES assumes parallel forms with identical factor structure, the use of alternate forms that are not exactly parallel merely results in a slight underestimation of the GCES (Le et al., 2009).
} 
evidence is "based on relationships with measures of other variables ... [and] test content" (SIOP, 2018, p. 9).

\section{Convergent Relations}

While past work on AVI-PAs provided some convergent evidence of validity, the work has focused solely on interviewer-reported personality and not self-reports (e.g., Biel et al., 2013; Chen et al., 2018; Ponce-Lopez et al., 2016). Yet, to maximize the utility of AVIs, it is critical to examine AVI-PAs trained on both self- and interviewer-reports. According to socioanalytic theory, self- and observer-reports (interviewer-reports being one example) represent two important aspects of personality: identity and reputation, respectively (Hogan, 1991). As mentioned earlier, the interviewers glean a context-specific reputation based on interviewee performance that corresponds closely to the behaviors used as inputs to AVI-PAs. However, prior AVI-PA research has not provided a clear rationale for focusing solely on interviewerreports. Theoretically, both self- and observer-reports are critical for understanding applicant personality, as each provides unique information about inward and outward expressions of personality traits, and practically, both are useful for predicting behavior (Connelly \& Ones, 2010). Therefore, the utility of AVI-PAs can be expanded if models trained on self-reported traits have substantial convergence, apart from models trained on interviewer-reports.

An important question at this point is what counts as substantial convergence? To address this question, we draw on automatic essay grading and its application to selection and assessment (Campion et al., 2016). Campion et al. (2016) applied text mining to automatically score achievement record essays and sought to develop a system that was at least as accurate as single raters, as indexed by single rater one-way random effects intraclass correlation (referred to as ICC(1) by McGraw \& Wong, 1996 and ICC (1, 1) by Shrout \& Fleiss, 1979). In their case, the 
average single rater one-way random effects intraclass correlation was .61, and their automatic scoring system converged, on average, $r=.63$ with human raters in the testing data. We adopt their metric and aim to develop AVI-PAs that converge as highly as single raters in our study. Research Question 2 involves replicating and extending prior work by investigating the convergent evidence of validity for machine learning models trained not just on interviewerreports but also on self-reports.

Research Question 2: Do AVI-PAs (trained on either self- or interviewer-reports) exhibit adequate convergence, as compared to single rater one-way random effects intraclass correlations?

\section{Discriminant Relations}

To our knowledge, there has not been any research examining AVIs' discriminant evidence of validity. This is a crucial psychometric property because validity will be questionable if test scores converge highly with measures of distinct constructs (Campbell \& Fiske, 1959). Indeed, a plausible source of construct-irrelevant variance is the assessment method itself (Messick, 1989). This systematic variance could inflate correlations between measures of purportedly distinct constructs to the point where they are empirically redundant (Raykov et al., 2016; Shaffer et al., 2016).

Concerningly, interview ratings often have substantial method variance, resulting in poor construct discrimination (Hamdani et al., 2014). Further, the machine learning models undergirding AVIs seek to maximize convergence with human ratings (Bleidorn \& Hopwood, 2019), similar to empirical keying. However, empirical keying often results in poor discriminant evidence (Simms, 2008). Park and colleagues (2015) examined the discriminant evidence for machine learning trait assessments derived from the language used in Facebook posts. The 
machine learning trait assessments exhibited inflated method variance compared to the selfreports on which the models were trained. Together, these concerns suggest a need to investigate the discriminant evidence of AVI-PAs.

Research Question 3: Do AVI-PAs exhibit adequate discriminant evidence?

\section{Generalizability Across Contexts}

In the case of AVIs, it is critical to determine whether a trained model generalizes to new samples for different interview contexts (Bleidorn \& Hopwood, 2019; Tay et al., 2020). This is because some AVI vendors train machine learning models on one or more sets of interview questions, then deploy them in hiring contexts where client organizations write new interview questions specific to the focal job. The Standards state that validity evidence should be provided for all intended uses and interpretations of a test, including any major alterations to tests, such as changing test questions (AERA et al., 2014). For example, language-based personality models trained on Twitter do not appear to generalize to transcriptions of mock interviews (Hickman et al., 2019). In the case of AVIs, they need to be similarly reliable and valid when they are applied to different interview questions. In addition to these practical concerns, behavior is a function of both the person and the situation (Mischel \& Shoda, 1995), even in interview contexts (Huffcutt et al., 2011). Therefore, the psychometric properties of AVIs must be investigated for crosssample predictions to justify their use.

Allowing organizations to supply job-relevant questions follows interview best practices (Campion et al., 1997). Still, it raises concerns that models trained on one set of interview questions will not generalize to new interviews. Prior studies of AVIs have focused on withinsample cross-validation, and to our knowledge, no evidence is available to suggest that the models generalize to new interview questions. In particular, verbal behavior likely differs 
between interviews because the interview questions exert considerable influence on what interviewees say. For instance, some evidence suggests that interviewees use more impression management tactics in behavioral (asking about prior actions) than situational (hypothetical scenario) interviews (Peeters \& Lievens, 2006). Therefore, different interview questions (i.e., situations) may cause differences in how interviewee personality relates to their behavior.

In the present study, each of the four samples of interviews involved different interview questions. Interview questions in Sample 1 were not intended to tap a particular set of constructs. Interview questions in Sample 2 were designed to tap constructs relevant to many jobs and some Big Five traits (i.e., leadership and teamwork). Finally, interview questions in Samples 3 and 4 were explicitly designed to tap the Big Five traits. Due to the effects of situations on behavior, machine learning models trained on one sample's set of interview questions may contain question-specific variance that is trait-irrelevant, resulting in models with psychometric properties that do not generalize to other interview questions.

Research Question 4: Do the psychometric properties of AVI-PAs trained on one set of interview questions generalize to different sets of interview questions? Specifically, does evidence generalize in terms of (a) test-retest reliability, (b) generalized coefficient of equivalence and stability, (c) convergent relations, and (d) discriminant relations?

\section{Content Coverage}

Another critical concern in AVIs is the content of the predictive models. Concerns have been raised, for example, that overreliance on nonverbal behavior in such models may discriminate against certain groups of applicants (EPIC, 2019; Harris et al., 2019). To assuage such concerns, some vendors have claimed that their assessments are mostly driven by what interviewees say (i.e., verbal behavior; Bell, 2019), yet those same vendors have, at times, made 
conflicting statements regarding the relative contributions of verbal, paraverbal, and nonverbal behavior to AVI assessments (cf. Harwell, 2019). Ickes (2016) summarized evidence that suggests all three types of behavior contribute to interpersonal judgment accuracy, with verbal behavior contributing the most information (50-60\%), followed by paraverbal behavior (approximately 30\%), and nonverbal behavior (10-20\%). Therefore, it is vital to explore the content of the models to understand the relative contribution of each type of behavior.

Although the relative contribution of verbal, paraverbal, and nonverbal behavior is one important element of AVI model content, it does not address whether the behaviors used in AVIPAs are related to the intended trait. Validity evidence based on test content involves examining the correspondence between test content and the construct it purports to measure (AERA et al., 2014). Therefore, it is critical to explore the predictors (i.e., interviewee behaviors) included in final models, for example, by exploring what predictors (if any) consistently emerge in sametrait models.

Research Question 5: In AVI-PAs, (a) how much weight is afforded to each type of behavior, and (b) what behavioral predictors (if any) are common to all same-trait models, and are they conceptually relevant to the focal trait?

\section{Nomological Network}

Another key metric for judging the utility of personality assessments is whether personality scores relate to other constructs and relevant behaviors in meaningful ways (Connelly \& Ones, 2010; Funder, 2012). For instance, conscientiousness predicts job performance across a wide range of jobs (Barrick \& Mount, 1991), and the remaining Big Five traits predict performance in jobs with relevant contextual demands (Judge \& Zapata, 2015).

In the present study, we collect self-reported academic performance and standardized test 
scores to provide initial evidence relating AVI-PAs to external variables known to be associated with Big Five traits. Relating AVI-PAs to standardized test scores and academic outcomes represents a first step toward investigating workplace relevant criteria. Theoretically, conscientiousness predicts greater persistence, and openness predicts intellectual exploration. Further, agreeableness leads to more interpersonally cooperative behavior, which could improve academic performance through participation and group work scores. Conscientiousness is strongly related to high school and college grade point average (GPA), while agreeableness and openness are weakly positively related to high school and college GPA (Noftle \& Robins, 2007; Poropat, 2009). Additionally, openness is positively related to SAT verbal test scores because open individuals acquire more vocabulary. Emotional stability and extraversion tend to be weakly and inconsistently related to academic outcomes. Therefore, we take initial steps toward examining the nomological network of AVI-PAs by regressing academic performance (i.e., high school and college GPA) and standardized test scores (i.e., SAT and ACT scores) on crosssample AVI-PAs.

Research Question 6: Do AVI-PAs correlate with academic outcomes, and do they do so incrementally beyond self- and interviewer-reported traits?

\section{Method}

\section{Sample 1}

The first sample consists of two groups of master-status Amazon Mechanical Turk workers (Turkers; total $\mathrm{N}=337 ; 58 \%$ female) who participated in open-ended interviews as part of an unrelated project by a private company. The first group was paid $\$ 1$ for participating, and the second group was paid $\$ 1$ for completing a survey and another $\$ 1$ when their video (and audio) submissions were confirmed. The two sets of open-ended questions represent relatively 
unstructured interviews (e.g., Blackman, 2002). The first set of 157 Turkers responded to the following prompt, "Talk about a topic or a story that you know and is personal to you. Do not hesitate to talk about your feelings and do not limit your answer to simple descriptions. Options include: 1. A personal experience (traveling, childhood memory, recent event). 2. Your dreams (career, love, friends, hobbies). 3. Your general views on a matter you feel strongly about." Hickman et al. (2019) used this first set of Turkers to investigate the validity of Twitter-based algorithm for assessing personality in the interview context. The second set of 179 Turkers completed a self-report personality inventory and responded to the following prompt, "1) Tell me about your dream job, and why you think you would be successful in this job? 2) Tell us about a time when things didn't go the way you wanted-like a promotion you wanted and didn't get, or a project that didn't turn out how you had hoped. Describe what approach you took to solve the problem." These Turkers were mostly employed (76.8\%) at the time of the study. Of the Turkers who were employed, $15.6 \%$ worked in education, $13.7 \%$ worked in health care, $12.2 \%$ worked in “other services (except public administration)," 10.8\% worked in professional, scientific, or technical services, $9.4 \%$ worked in retail, $7.2 \%$ worked in arts, entertainment, or recreation, and the remaining industry groups each comprised less than $5 \%$ of the sample. Across the two groups, the average interview length was $3 \min 8 \mathrm{~s}$ and 404 words. After the data was cleaned and shared with the research team, two participants were dropped because they read responses directly from a website. Additionally, due to technical issues in extracting verbal, paraverbal, or nonverbal behavior (e.g., poor camera or audio quality), a further 11 participants were excluded, resulting in a final sample $\mathrm{N}=324$, with self-report $\mathrm{N}=170$. Reliability information for all selfand interviewer-reported traits is provided in the Results section.

\section{Sample 2}


We recruited 490 undergraduate students (50\% female) who participated in the study for course credit (Purdue University IRB protocol 1806020758, Automated Assessment of Candidate Interviews). Participants were randomly assigned to complete the study remotely or proctored in the lab to maximize the number of interviews conducted. Participants self-reported their personality traits. Then, each participant recorded three 2-3 minute videos, one video for each of the following prompts, "Please tell us about yourself," "Please tell us about a time you demonstrated leadership," and "Please tell us about a time you worked effectively in a team." The average total interview length was 6 min $51 \mathrm{~s}$ and 951 words. Four hundred sixty-seven participants completed the study in full. Thirty-five interviews were unusable due to technical difficulties experienced during the study (e.g., no sound), leaving a total of $\mathrm{N}=432$. Fifty participants' self-reported traits were excluded for failing one or more attention check questions, leaving $\mathrm{N}=382$.

\section{Sample 3}

We recruited 361 undergraduate students (52\% female) who participated in the study for course credit (Purdue University IRB protocol 18110213366, Automatically Assessing

Behavioral and Situational Interviews). Participants were randomly assigned to conditions of a 2 (location: remote interview versus proctored in lab interview) x 2 (question type: past behavioral versus situational questions) factorial design. The remote condition was again used to maximize the amount of data collected, and changing the question type was done to reduce the influence of the situation on the resulting models by sampling from multiple situations (but neither condition was treated as an independent variable in the present study). All participants self-reported personality traits. Then, each participant recorded five 1-3 minute videos, one video for each of the prompts designed to tap into the Big Five traits. For those who received behavioral interview 
questions, the prompts were: "Think of a time you had a need to learn about something that was new to you? Why did you pursue it? What kept you persistent?" (openness); "Think of a time a coworker asked you to set aside your own work to help him or her with a project that was very important to them. What did you do? Why did you do that?" (agreeableness); "Tell me about a recent uncomfortable or difficult work situation. How did you approach this situation? What happened?" (emotional stability); "Tell me about a situation when you had to speak up in order to get a point across that was important to you or crucial to your customer. How did you go about this?" (extraversion); and "Describe a long-term project that you managed. What did you do to keep everything moving along in a timely manner?” (conscientiousness). To turn the past behavioral questions into situational questions, we asked interviewees to "imagine" that the scenarios posed in the questions occurred, and asked them "what would you do," following prior research (e.g., Van Iddekinge et al., 2005). The average total interview length was 7 min $29 \mathrm{~s}$ and 883 words. Forty-four participants were dropped because their videos were unusable due to technical difficulties, leaving a total of $\mathrm{N}=317$. A further 28 participants' self-reports were dropped for failing one or more attention check questions, leaving $\mathrm{N}=289$. A subset of these participants $(\mathrm{N}=110)$ gave us permission to share their video recordings on Databrary, which are available at http://doi.org/10.17910/b7.1171

\section{Sample 4}

We recruited 101 undergraduate students (43\% female) who participated twice in the mock interview for course credit (Purdue University IRB protocol 1907022479, Advancing Video Interviews Toward Computerized Assessment). Participants completed the study in the lab, and they answered the same interview questions on both occasions. The first administration occurred one to 93 days prior to the second administration, with an average of 15.6 days (median 
= 11 days) between them. Each participant recorded six 1-3 minute videos, one each for the five behavioral questions in Sample 3, as well as, "Think of a time you were a member of a successful team. Describe the role you played on the team and in its success." Two participants were dropped because their videos were unusable due to technical difficulties, leaving a final sample size $\mathrm{N}=99$. The data from Samples 2, 3, and 4 are part of broader data collection efforts.

Although we know less about the motivation of Turkers in Sample 1, in all three samples of students, students appeared to consider these mock interviews as a serious opportunity to improve their interviewing skills. Many students either took considerable time preparing their responses before recording them or re-recorded their answers (sometimes upwards of three times) to improve the impression they would make.

\section{Measures}

\section{Self-reported Personality}

The second group of Sample 1 Turkers responded to a 60 -item composite of the 50-item Big Five markers (Goldberg, 1992) and the ten-item personality inventory (TIPI; Gosling et al., 2003) using a 7-point Likert scale. The first group of Turkers in Sample 1 did not self-report their personality traits. Participants in Samples 2 and 3 responded to the 50-item Big Five markers obtained from the IPIP (Goldberg, 1992, 1999) using a five-point Likert scale.

\section{Interviewer-reported Personality}

For Sample 1, three Industrial-Organizational Psychology Ph.D. students watched and rated each interviewee on the TIPI $(\mathrm{N}=324)$. For Sample 2, at least four undergraduate research assistants from a pool of eleven (range: 4-7) watched and rated each interviewee on the TIPI (N = 432). For Sample 3, four undergraduate research assistants watched and rated each interviewee on the TIPI $(\mathrm{N}=317)$. In all three cases, raters underwent 1-2 hours of frame of reference 
training that consisted of defining the Big Five traits, reviewing the scale items and response format, conducting practice ratings, and discussing ways of interpreting interviewee behavior in relation to Big Five traits. Participants who failed attention checks had their self-reports excluded but were included in the interviewer-reports to maximize sample size.

\section{Self-reported Academic Performance}

In Samples 2 and 3, participants self-reported their high school grade point average (GPA) and college GPA. Additionally, participants self-reported their SAT verbal, SAT math, and ACT scores if they took one or both tests. Self-reported academic outcomes converge to a high degree $(r>.8)$ with actual scores (Kuncel et al., 2005). We excluded the college GPAs of students in their first semester of college.

\section{Verbal Behavior}

Participant responses were transcribed using IBM Watson Speech to Text (IBM, 2019), and their full interview response was combined into a single document. Then, we first used Linguistic Inquiry and Word Count (LIWC; Pennebaker et al., 2015) to quantify verbal behavior. We used all directly counted non-punctuation variables from LIWC, including word count. However, after inspecting the data, we noticed that several LIWC categories that are likely irrelevant to employment interviews tended to have very low base rates, and therefore, low variability, so these variables were not included in subsequent analyses (i.e., death, netspeak, cursewords, and fillers).

Second, we used the $t m \mathrm{R}$ package (Feinerer et al., 2008) and procedures described in Speer (2018) to count one- and two-word phrases (i.e., $n$-grams with $\mathrm{n}=1$ or 2 ) in the text. Before extracting the words and phrases, we first removed all numbers and punctuation from the transcripts, removed common stop words, transformed all text to lowercase, handled negation by 
appending words preceded by "not," "n't," "cannot," "never," and "no" with the negator and an underscore, and stemmed the corpus. We removed all one- and two-word phrases that did not occur in at least $2 \%$ of the interviews. The resulting document-term matrices were populated by the counts of the remaining words and phrases (e.g., if job occurred five times in a participant's response, it was assigned a value of 5 for that participant).

\section{Paraverbal Behavior}

We extracted paraverbal behaviors with openSMILE (Eyben, 2014; Eyben et al., 2016), which was developed using multiple baseline feature sets to train the system to measure acoustic features. We utilized openSMILE to extract a common, relatively low-dimensional set of features called the Geneva Minimalistic Acoustic Parameter Set (Eyben et al., 2016). These features included pitch, indices of voice quality like jitter and harmonics-to-noise ratio, frequency, loudness, speech rate, and more, as detailed in Table 1. We extracted features in overlapping 30-second windows of time, sliding windows in 1-second steps, then aggregated the results using means, standard deviations, skewness, and kurtosis.

\section{Nonverbal Behavior}

Recently, concerns have been raised over the use of emotion analytics software that extracts discrete facial emotions (Barrett et al., 2019). For example, facial expressions may be heavily influenced by context, such as social pressure to appear calm while stressed during an interview. Therefore, we used the raw features described by the software rather than the emotionlevel abstractions to avoid a priori assumptions about the context-specific interpretation of particular facial expressions. OpenFace describes 19 facial action units (AUs) in addition to head pose features (Baltrušaitis et al., 2018). For each facial action unit, we calculated the mean intensity, as well as its standard deviation, kurtosis, and skewness. Additionally, we extracted 
cooccurrence distributions (Bosch \& D’Mello, 2019) for each pair of AUs, represented as the distribution similarity between two AUs measured via Jensen-Shannon divergence (Lin, 1991). Cooccurrence distributions represent the degree to which two AUs activate in similar ways within a video. Cooccurrences capture more complex facial expressions than individual AUs. For example, similar distributions for smile and eye-related AUs may differentiate genuine (i.e., Duchenne) smiles from acted smiles wherein the mouth moves independently of the muscles around the eyes (Messinger et al., 2001). Finally, we also extracted head pose information along vertical (yaw), horizontal (pitch), and depth (roll) dimensions, again using the mean, standard deviation, kurtosis, and skewness to describe these features.

\section{Analytic Strategy}

\section{Model Development and Within-Sample Validation}

In total, we trained 30 predictive models using elastic net regression to predict selfreported and interviewer-reported [2] Big Five traits [5] in three datasets [3] $(2 \times 5 \times 3=30)$. In each case, we used the verbal, paraverbal, and nonverbal behavior indicators listed in Table 1 as predictors, and all these predictors were calculated using the entire interview response (i.e., at the interviewee-level). Within each sample for each trait, we used the caret R package (Kuhn, 2008) and a loop to conduct nested $k$-fold cross-validation with $k=10$ for both the inner and outer folds. Figure 2 illustrates this process, and the Online Supplement includes the code we used to conduct nested cross-validation.

Figure 2

Nested cross-validation where $k=10$ for inner and outer folds 


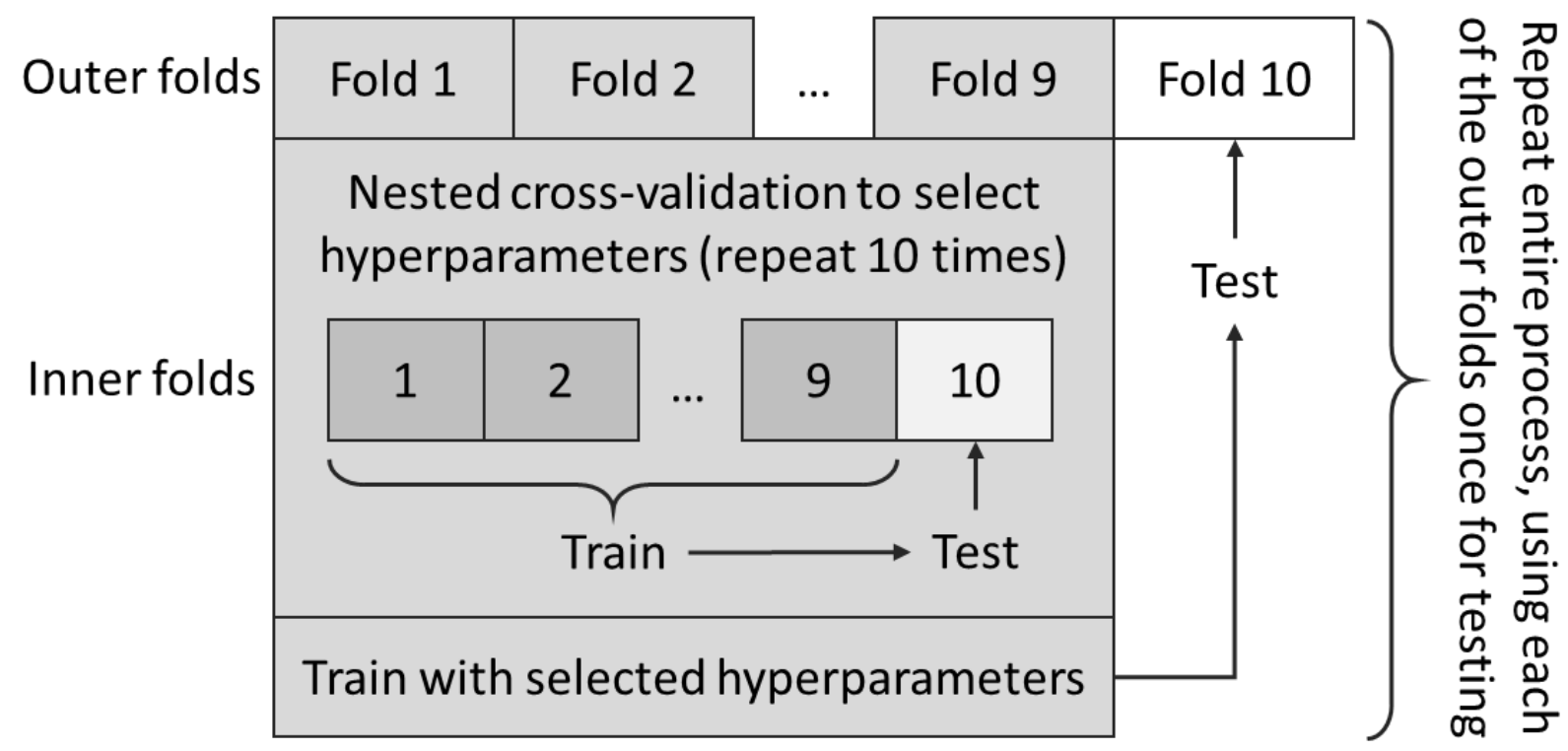

Nested cross-validation with $k=10$ involves splitting the data into ten equally sized parts (the outer folds). Then, nine of these parts (the outer training folds) are used to conduct a separate 10-fold (the inner folds) cross-validation to select the optimal elastic net hyperparameters (i.e., model selection) based solely on these nine outer folds. Next, the final model is trained on those nine folds using the optimal hyperparameters, and then that model's accuracy is estimated on the outer test fold (i.e., model assessment). This process is repeated 10 times, using each of the ten outer folds only once for testing. $k$-fold cross-validation balances model bias and variance to maximize out-of-sample accuracy (Chapman et al., 2016; Putka et al., 2018), and nested cross-validation ensures that information about model accuracy is not used for hyperparameter tuning, reducing the likelihood that accuracy will be overestimated. Within selfand interviewer-reports in each sample, the composition of the 10 outer folds was held constant across the five traits so that, for example, the first test fold for the extraversion model contained the same participants as the first test fold for the other four trait models.

The predictions from each of the final elastic net models were then correlated with the traits they were trained to predict (i.e., self- or interviewer-reported) in each test fold to generate 
multi-trait multimethod correlation matrices. These correlations were averaged across the ten test folds to examine within-sample convergent- and discriminant relations. The average of the correlation matrices from each test fold was used because, in nested 10-fold cross-validation, a separate model is trained and tested for each test fold, each with its own set of hyperparameters, which is, essentially, ten replications of the same procedure. Calculating model performance by averaging across the test folds in $k$-fold cross-validation follows the practices of two of the most popular software packages for machine learning (Kuhn, 2008; Pedregosa et al., 2011).

\section{Cross-sample Trait Assessments and Validation}

We trained separate models on each of the full sets of participants in Samples 1, 2, and 3. The optimal hyperparameters for these models were identified separately for each sample via 10fold cross-validation, and the trained models were applied to assess self- and interviewerreported Big Five traits in the other three samples (i.e., Sample 1 models were applied to Samples 2, 3, and 4; Sample 2 models were applied to Samples 1, 3, and 4; Sample 3 models were applied to Samples 1, 2, and 4). Concerning reliability, we applied trained models derived from Samples 1, 2, and 3 to Sample 4 (test-retest sample). To calculate overall test-retest reliability, we did the following: (a) for each model derived from Samples 1, 2, and 3, we applied them to assess traits at each of the two time points in Sample 4; and (b) we calculated the testretest correlation for each trait for scores from each of the models derived from Samples 1, 2, and 3. We calculated GCES by averaging the alternate forms (i.e., models derived from different samples; Sample $1 \&$ 2; Sample $2 \& 3$; Sample $1 \&$ 3) time 1-time 2 same-trait correlations in Sample 4. For instance, to estimate GCES $\mathrm{s}_{\mathrm{s} s 2}$ for Sample 1 and 2 extraversion models, we averaged (a) the correlation of Sample 1 models' Time 1 extraversion scores with Sample 2 models' Time 2 extraversion scores and (b) the correlation of Sample 2 models' Time 1 
extraversion scores with Sample 1 models' Time 2 extraversion scores. Because we had three sets of trait assessments, we averaged all three alternate form pairs to obtain overall GCES for extraversion.

With regard to validity, we applied the trained models derived from Samples 1, 2, and 3 to predict self- and interviewer-reported traits in the other two samples (e.g., Sample 1 models were applied to Samples 2 and 3). We calculated multitrait-multimethod matrices using the trait predictions and the traits they were trained to predict to analyze convergent and discriminant relations.

To explore the content of AVI-PAs, we generated standardized regression weights by standardizing the predictors (i.e., by subtracting each predictor's mean and dividing by the standard deviation) in Samples 1, 2, and 3 before training models on the full set of participants in each sample. Then, we separately summed the standardized regressions weights for verbal behavior predictors, paraverbal behavior predictors, and nonverbal behavior predictors and divided each by the total sum of regression weights to estimate the relative contribution of each type of behavior to the final models.

Finally, to inspect the nomological network of AVI-PAs, we first examined the bivariate correlations between AVI-PAs and academic outcomes. Then, we regressed academic outcomes in Samples 2 and 3 onto the cross-sample trait predictions (e.g., Sample 2 ACT scores were regressed separately onto the Sample 2 trait scores from models trained on Samples 1 and 3).

\section{Elastic Net Regression}

Elastic net regression is a hybrid of ridge and least absolute shrinkage and selection operator (LASSO) regression (Zou \& Hastie, 2005). It has two hyperparameters. One hyperparameter determines whether elastic net acts more like ridge regression, by shrinking 
regression coefficients toward zero in response to collinearity and model complexity, or more like LASSO, by shrinking regression coefficients to zero (i.e., performing variable selection). The second hyperparameter determines how severely regression weights are penalized. We determined the optimal hyperparameters by searching through 10 values of each during $k$-fold cross-validation. Elastic net is useful when the $n$-to- $p$ ratio (i.e., ratio of the number of observations to the number of predictors) is low because it reduces model complexity (e.g., Oswald et al., 2020; Speer, 2018).

\section{Results}

\section{Descriptive Statistics of Self- and Interviewer-Reports}

Tables 3, 4, and 5 present descriptive statistics, intercorrelations, and reliabilities for selfand interviewer-reported traits in each of the first three samples. Tables 4 and 5 also include correlations with academic outcomes. In all cases, the convergent correlations between self- and interviewer-reported traits were positive, and in most cases, the convergent correlations were significant. Self-other agreement was lowest for conscientiousness $(\bar{r}=.09)$, followed by openness $(\bar{r}=.15)$, and emotional stability $(\bar{r}=.20)$. Extraversion had the highest level of selfother convergence $(\bar{r}=.33)$, followed by agreeableness $(\bar{r}=.30)$. With the exception of agreeableness, these findings largely align with the RAM (Funder, 1995)—self-other convergence will be lower for more evaluative traits where self-reports may be less accurate, like conscientiousness, as well as for internal, less-visible traits where interviewer-reports may be less accurate, like openness and emotional stability. Overall, self-report reliabilities were acceptable (Sample $1 \alpha$ range: $.83-.93, M=.89$; Sample $2 \alpha$ range: .76, $.90, M=.83$; Sample $3 \alpha$ range: $.76-.89, M=.82)$ and the interrater reliabilities met or exceeded .60 for all traits except emotional stability in Sample 2 and agreeableness, emotional stability, and openness in Sample 3 
Table 3

Sample 1: Correlation matrix of self-and interviewer-reported personality

\begin{tabular}{|c|c|c|c|c|c|c|c|c|c|c|c|c|}
\hline Variable & $M$ & $S D$ & 1 & 2 & 3 & 4 & 5 & 6 & 7 & 8 & 9 & 10 \\
\hline \multicolumn{13}{|l|}{ Self-reports } \\
\hline 1. Extraversion & 4.02 & 1.27 & $(.92)$ & & & & & & & & & \\
\hline 2. Agreeableness & 5.53 & 0.90 & $.31 * *$ & $(.86)$ & & & & & & & & \\
\hline 3. Conscientiousness & 5.05 & 1.09 & .11 & $.21 * *$ & $(.90)$ & & & & & & & \\
\hline 4. Emotional stability & 4.21 & 1.35 & $.35 * *$ & $.29 * *$ & $.53 * *$ & $(.93)$ & & & & & & \\
\hline 5. Openness & 5.49 & 0.81 & $.33 * *$ & $.45 * *$ & $.26 * *$ & $.15^{*}$ & $(.83)$ & & & & & \\
\hline \multicolumn{13}{|l|}{ Interviewer-reports } \\
\hline 6. Extraversion & 4.27 & 1.17 & $.22 * *$ & .14 & .01 & .13 & $.19^{*}$ & $(.76)$ & & & & \\
\hline 7. Agreeableness & 4.62 & 0.91 & .09 & .13 & .04 & .02 & -.09 & $.21 * *$ & $(.74)$ & & & \\
\hline 8. Conscientiousness & 4.74 & 0.93 & .10 & .03 & .07 & .11 & .06 & $.21 * *$ & $.34 * *$ & $(.80)$ & & \\
\hline 9. Emotional stability & 4.66 & 0.95 & $.19^{*}$ & .05 & $.22 * *$ & $.29 * *$ & .05 & $28 * *$ & $.33 * *$ & $.44 * *$ & $(.63)$ & \\
\hline 10. Openness & 4.26 & 0.97 & $.16^{*}$ & .04 & -.14 & -.07 & .13 & $.26 * *$ & $.25^{* *}$ & $.19 * *$ & .11 & $(.67)$ \\
\hline
\end{tabular}

Note. Interviewer-report $\mathrm{N}=324$. Self-report $\mathrm{N}=170 . M$ and $S D$ are used to represent mean and standard deviation, respectively. $*$ indicates $p<.05$. ** indicates $p<.01$. Reliabilities reported in diagonal-for self-reports, Cronbach's alpha, and for interviewerreports, $\operatorname{ICC}(2, k)$. 
Table 4

Sample 2: Correlation matrix of self-and interviewer-reported personality and self-reported academic outcomes

\begin{tabular}{|c|c|c|c|c|c|c|c|c|c|c|c|c|c|c|c|c|}
\hline Variable & $M$ & $S D$ & 1 & 2 & 3 & 4 & 5 & 6 & 7 & 8 & 9 & 10 & 11 & 12 & 13 & 14 \\
\hline \multicolumn{17}{|l|}{ Self-reports } \\
\hline 1. Extraversion & 3.18 & 0.84 & $(.90)$ & & & & & & & & & & & & & \\
\hline 2. Agreeableness & 4.03 & 0.59 & $.29 * *$ & $(.83)$ & & & & & & & & & & & & \\
\hline 3. Conscientiousness & 3.54 & 0.63 & $.11 *$ & $.14 * *$ & $(.80)$ & & & & & & & & & & & \\
\hline 4. Emotional stability & 3.01 & 0.72 & $.15^{* *}$ & .02 & $.14^{*}$ & $(.84)$ & & & & & & & & & & \\
\hline 5. Openness & 3.68 & 0.55 & $.21 * *$ & $.25 * *$ & $.11 *$ & .03 & $(.76)$ & & & & & & & & & \\
\hline \multicolumn{17}{|l|}{ Interviewer-reports } \\
\hline 6. Extraversion & 4.55 & 1.16 & $.41 * *$ & $.28 * *$ & .08 & -.02 & .07 & $(.89)$ & & & & & & & & \\
\hline 7. Agreeableness & 4.80 & 0.70 & $.14 *$ & $.43 * *$ & .06 & -.02 & .02 & $.29 * *$ & $(.62)$ & & & & & & & \\
\hline 8. Conscientiousness & 5.45 & 0.62 & .02 & .07 & .04 & -.03 & .04 & $.29 * *$ & $.14 * *$ & $(.60)$ & & & & & & \\
\hline 9. Emotional stability & 5.09 & 0.62 & $.21 * *$ & $.18 *$ & $.11 *$ & $.11 *$ & .04 & $.40 * *$ & $.24 * *$ & $.35 * *$ & $(.58)$ & & & & & \\
\hline 10. Openness & 4.56 & 0.87 & .12 & $.19 * *$ & $-.16 * *$ & -.07 & $.17 * *$ & $.43 * *$ & $.17 * *$ & $.38 * *$ & $.36 * *$ & $(.72)$ & & & & \\
\hline \multicolumn{17}{|l|}{ Academic Outcomes } \\
\hline 11. College GPA & 3.35 & 0.48 & -.14 & -.01 & .08 & -.13 & -.04 & -.04 & -.03 & $.15^{*}$ & -.13 & -.09 & & & & \\
\hline 12. High school GPA & 3.75 & 0.26 & -.05 & $.15 * *$ & $.22 * *$ & .02 & $.12^{*}$ & $.15 * *$ & $.15 * *$ & .09 & .04 & .10 & $.20 * *$ & & & \\
\hline 13. SAT Verbal & 628.0 & 79.0 & -.08 & -.04 & -.01 & -.06 & $.19 * *$ & .04 & -.04 & $.17 *$ & -.02 & $.15^{*}$ & .08 & $.18 * *$ & & \\
\hline 14. SAT Math & 645.3 & 102 & $-.18 * *$ & $-.24 * *$ & -.02 & .00 & .02 & -.08 & $-.24 * *$ & $.24 * *$ & -.04 & .10 & $.18 * *$ & $.16^{* *}$ & $.50 * *$ & \\
\hline 15. ACT & 27.90 & 4.02 & -.13 & -.02 & .05 & .01 & .15 & .09 & -.05 & $.17 * *$ & .10 & $.29 * *$ & .03 & $.33 * *$ & $.52 * *$ & $.46 * *$ \\
\hline
\end{tabular}

Note. Interviewer-report $\mathrm{N}=432$. Self-report $\mathrm{N}=382$. College GPA N $=161$. High school GPA $\mathrm{N}=375$. SAT verbal $\mathrm{N}=302 . \mathrm{SAT}$ math $\mathrm{N}=306$. ACT $\mathrm{N}=222 . M$ and $S D$ are used to represent mean and standard deviation, respectively. $*$ indicates $p<.05$. $* *$ indicates $p<.01$. Reliabilities reported in diagonal—for self-reports, Cronbach's alpha, and for interviewer-reports, ICC $(1, k)$. 
Table 5

Sample 3: Correlation matrix of self-and interviewer-reported personality and self-reported academic outcomes

\begin{tabular}{|c|c|c|c|c|c|c|c|c|c|c|c|c|c|c|c|c|}
\hline Variable & $M$ & $S D$ & 1 & 2 & 3 & 4 & 5 & 6 & 7 & 8 & 9 & 10 & 11 & 12 & 13 & 14 \\
\hline \multicolumn{17}{|l|}{ Self-reports } \\
\hline 1. Extraversion & 3.34 & 0.82 & (.89) & & & & & & & & & & & & & \\
\hline 2. Agreeableness & 4.02 & 0.60 & $.42 * *$ & $(.83)$ & & & & & & & & & & & & \\
\hline 3. Conscientiousness & 3.53 & 0.59 & .10 & $.13^{*}$ & $(.76)$ & & & & & & & & & & & \\
\hline 4. Emotional stability & 3.05 & 0.81 & $.20 * *$ & -.03 & $.24 * *$ & $(.87)$ & & & & & & & & & & \\
\hline 5. Openness & 3.68 & 0.55 & $.27 * *$ & $.29 * *$ & $.15^{* *}$ & $.16^{* *}$ & $(.76)$ & & & & & & & & & \\
\hline \multicolumn{17}{|l|}{ Interviewer-reports } \\
\hline 6. Extraversion & 4.43 & 1.16 & $.30 * *$ & $.28 * *$ & .09 & -.04 & $.15^{*}$ & $(.78)$ & & & & & & & & \\
\hline 7. Agreeableness & 4.96 & 0.70 & $.24 * *$ & $.34 * *$ & $.12 *$ & .04 & $.15^{*}$ & $.30^{* *}$ & $(.58)$ & & & & & & & \\
\hline 8. Conscientiousness & 5.30 & 0.70 & .02 & .08 & $.15 *$ & .03 & $.14 *$ & $.27 * *$ & $.34 * *$ & $(.65)$ & & & & & & \\
\hline 9. Emotional stability & 5.09 & 0.63 & .13 & $.13^{*}$ & .09 & $.20 * *$ & $.17 * *$ & $.20 * *$ & $.42 * *$ & $.45^{* *}$ & $(.51)$ & & & & & \\
\hline 10. Openness & 4.34 & 0.78 & .16 & $.23 * *$ & .08 & -.01 & $.16^{* *}$ & $.45^{* *}$ & $.34 * *$ & $.41 * *$ & $.20 * *$ & (.49) & & & & \\
\hline \multicolumn{17}{|l|}{ Academic Outcomes } \\
\hline 11. College GPA & 3.15 & 0.61 & .01 & -.02 & $.20 * *$ & .05 & -.07 & .09 & $.21 * *$ & $.19 *$ & .06 & $.17 *$ & & & & \\
\hline 12. High school GPA & 3.71 & 0.26 & .00 & .13 & $.25 * *$ & .01 & .01 & .06 & .12 & .10 & .03 & .05 & $.28 * *$ & & & \\
\hline 13. SAT Verbal & 626.5 & 74.2 & -.01 & -.04 & .06 & .02 & $.27 * *$ & .10 & .03 & $.19 * *$ & .13 & .01 & .13 & .15 & & \\
\hline 14. SAT Math & 641.6 & 102 & -.01 & $-.21 * *$ & .00 & .15 & .09 & -.06 & -.16 & .08 & -.04 & -.03 & $.22 * *$ & .10 & $.37 * *$ & \\
\hline 15. ACT & 27.22 & 4.37 & .05 & .10 & .15 & .15 & $.30 * *$ & .10 & .12 & $.23 *$ & $.21 *$ & .10 & $.20 *$ & .15 & $.61 * *$ & $.53 * *$ \\
\hline
\end{tabular}

Note. Interviewer-report $\mathrm{N}=317$. Self-report $\mathrm{N}=289$. College GPA $\mathrm{N}=270$. High school GPA $\mathrm{N}=282 . \mathrm{SAT}$ verbal $\mathrm{N}=225 . \mathrm{SAT}$ math $\mathrm{N}=226$. ACT $\mathrm{N}=162 . M$ and $S D$ are used to represent mean and standard deviation, respectively. $*$ indicates $p<.05 . * *$ indicates $p<.01$. Reliabilities reported in diagonal—for self-reports, Cronbach's alpha, and for interviewer-reports, ICC $(2, k)$. 
(Sample 1 ICC $(2, k)$ range: .63-.80, $M=.72$; Sample 2 ICC $(1, k)$ range: $.58-.89, M=.68$; Sample $3 \mathrm{ICC}(2, k)$ range: $.49-.78, M=.60)$.

\section{Within-Sample Nested Cross-Validation}

The first step in developing our predictive algorithms involved nested cross-validation, using 10 outer folds for testing and 10 inner folds for hyperparameter tuning. In nested 10-fold cross-validation, 10 iterations of 10-fold cross-validation are conducted using nine folds of the data, and the accuracy of the resulting models is tested on the remaining fold. The models used for analysis are those with the hyperparameters that maximized cross-validated accuracy during the inner 10-fold cross-validation. Appendix Table A1 reports the within-sample cross-validated convergence in the outer test folds by trait for both self- and interviewer-report models.

\section{Convergent and Discriminant Relations}

To address Research Questions 2 and 3 (Research Question 1 is addressed later with Research Question 4), we summarize multitrait-multimethod (MTMM; Campbell \& Fiske, 1959) matrices using convergence, discrimination, and method variance indices (Woehr et al., 2012) in Table 6 for each set of outer 10-fold cross-validated AVI-PAs and the corresponding self- or interviewer-reports. The convergence index (C1) is the average of the monotrait-heteromethod correlations. C1 should be positive and sufficiently large to show convergence, a necessary condition for further exploration of validity (Woehr et al., 2012). The first discrimination index (D1) indicates the amount of variance attributable to traits by comparing $\mathrm{C} 1$ to the average magnitude of heterotrait-heteromethod correlations. The second discrimination index (D2) indicates the amount of variance attributable to traits by comparing $\mathrm{C} 1$ to the average magnitude of heterotrait-monomethod correlations. D1 and D2 should be positive, and higher values suggest better construct discrimination. Method variance (MV) is calculated by comparing the average 
magnitude of heterotrait-monomethod correlations to the average magnitude of heterotrait-

heteromethod correlations. Higher values indicate greater method variance.

Table 6

Multitrait-multimethod statistics for AVI-PAs during within-sample cross-validation

\begin{tabular}{lcccccc}
\hline & $\mathrm{C} 1$ & $\mathrm{D} 1$ & $\mathrm{D} 2$ & $\mathrm{MV}$ & $\mathrm{D} 2 \mathrm{a}$ & $\mathrm{MV}_{\mathrm{a}}$ \\
\hline Van Iddekinge et al. (2005) & .32 & .12 & -.11 & .23 & -.25 & .37 \\
Park et al. (2015) & .38 & .27 & .15 & .11 & .10 & .16 \\
Sample 1 Self-report Models & .06 & .00 & -.15 & .15 & -.03 & .03 \\
Sample 2 Self-report Models & .11 & .02 & -.02 & .04 & -.01 & .03 \\
Sample 3 Self-report Models & .19 & .11 & .03 & .08 & .06 & .05 \\
Sample 1 Interviewer-report Models & .41 & .23 & .16 & .07 & .17 & .06 \\
Sample 2 Interviewer-report Models & .42 & .23 & .10 & .13 & .09 & .14 \\
Sample 3 Interviewer-report Models & .38 & .15 & .02 & .13 & .00 & .15 \\
\hline
\end{tabular}

Note: $\mathrm{C} 1$ = convergence index (average of monotrait-heteromethod correlations). D1 = discrimination index 1 (average of monotrait-hetermethod correlations minus the average magnitude of heterotrait-heteromethod correlations). D2 = discrimination index 2 (average of monotrait-heteromethod correlations minus the average magnitude of heterotrait-monomethod correlations). $\mathrm{MV}=$ method variance (average magnitude of heterotrait-monomethod correlations minus the average magnitude of heterotrait-heteromethod correlations). D2 $\mathrm{a}=$ discrimination index 2 calculated using only automated video interviews' heterotraitmonomethod correlations. $\mathrm{MV}_{\mathrm{a}}=$ method variance for automated video interviews.

For self-report models, $\mathrm{C} 1$ averaged $M=.12$ and ranged from .06 (Sample 1) to .19 (Sample 3). D1 averaged $M=.04$ and ranged from .00 (Sample 1) to .11 (Sample 3), and D2 averaged $M=-.05$ and ranged from -.15 (Sample 1) to .03 (Sample 3). MV averaged $M=.09$ and ranged from .04 (Sample 2) to .15 (Sample 1). D2a averaged $M=.01$ and ranged from -.03 (Sample 1) to .06 (Sample 3), and $\mathrm{MV}_{\mathrm{a}}$ averaged $M=.03$ and ranged from .03 (Sample 1) to .05 (Sample 3). None of the models trained on self-reports exhibited strong convergent relations, and the remaining indices only satisfied Woehr et al.'s (2012) criteria for the models from Sample 3.

For interviewer-report models, $\mathrm{C} 1$ averaged $M=.40$ and ranged from .38 (Sample 3 ) to .42 (Sample 2). D1 averaged $M=.20$ and ranged from .15 (Sample 3) to .23 (Samples $1 \& 2$ ), and D2 averaged $M=.09$ and ranged from .02 (Sample 3) to .16 (Sample 1). MV averaged $M=$ .11 and ranged from .07 (Sample 1) to .13 (Samples $2 \& 3$ ). D2 averaged $M=.09$ and ranged 
from .00 (Sample 3) to .17 (Sample 1), and $\mathrm{MV}_{\mathrm{a}}$ averaged $M=.12$ and ranged from .06 (Sample 1) to .15 (Sample 3). Of the models trained on interviewer-reports, all three showed appropriate patterns of convergent and discriminant relations, but the evidence was weakest for the Sample 3 models and strongest for the Sample 1 models.

As can be seen in Table 6, none of the self-report models exhibited C1 or D1 superior to Van Iddekinge et al.'s (2005) results. On the other hand, all three sets of interviewer-report models exhibited superior convergent and discriminant evidence of validity compared to Van Iddekinge et al.'s (2005) results. Further, the interviewer-report models from Samples 1 and 2 exhibited convergent and discriminant evidence of validity comparable or superior to Park et al.'s (2015) language-based personality models trained on nearly 70,000 self-reports.

Notably, however, the summary indices mask considerable variation among traits in terms of convergence, as summarized in Appendix Table A1. To judge the level of convergence, we consider the average of the single rater one-way random effects intraclass correlations from interviewer-reports - in Sample 1, the average ICC $(1,1)=.46$ (however, in Sample 3, the average ICC $(1,1)=.27)$. In self-report models, conscientiousness was least accurately assessed $(\bar{r}=.01)$, and emotional stability was most accurately assessed $(\bar{r}=.21)$. The models trained on self-reports in Sample 3 conform to the predictions of SOKA, as emotional stability and openness were most accurately inferred ( $r s=.31$ and .25 respectively in Sample 3), whereas extraversion and agreeableness were most accurately inferred in Sample $2(r \mathrm{~s}=.30$ and .25 , respectively). However, in no case did the convergence of self-report models exceed ICC $(1,1) \mathrm{s}$ from Sample 1.

In interviewer-report models, emotional stability was least accurately assessed $(\bar{r}=.27)$, and extraversion was most accurately assessed $(\bar{r}=.65)$. Across all three samples, the 
convergence of extraversion predictions exceeded ICC $(1,1)$ values. In Samples 1 and 2, the convergence of agreeableness was comparable to $\operatorname{ICC}(1,1)(\bar{r}=.43)$, but Sample 3 was considerably lower $(r=.17)$. Across all three samples, the convergence of conscientiousness models was comparable to $\operatorname{ICC}(1,1)(\bar{r}=.42)$, and the convergence of emotional stability models was lower than $\operatorname{ICC}(1,1)(\bar{r}=.27)$. The convergence of Sample 3's openness model was comparable to $\operatorname{ICC}(1,1)(r=.41)$, while the convergence of Samples 1 and 2's openness models were somewhat lower ( $r s=.27$ and .37 , respectively). Overall, there is evidence that AVI-PAs trained on interviewer-reports can converge at least as high as a single interviewer for some traits. The evidence is by far the strongest for extraversion models.

On average, interviewer-report models had higher convergence than self-report models $(\bar{r}$ $=.41$ vs. $\bar{r}=.10 ; z=7.3$, two-tailed $p<.01$ for Fisher $r$-to- $z$ transformation). However, the difference was not significant for emotional stability $(\bar{r}=.21$ vs. $\bar{r}=.27 ; z=1.38, p=.17)$. We now turn to examine how well the psychometric properties of AVI-PAs generalized to new interviews by investigating their reliability, replicating and extending the convergent and discriminant evidence provided within each sample for cross-sample assessments in new interviews, exploring AVI-PA model content, and examining their nomological network with regard to academic outcomes.

\section{Cross-Sample Generalizability of Automated Video Interview Trait Assessments}

To examine Research Question 4, we trained models on the full data available in each sample using the optimal hyperparameters identified by 10 -fold cross-validation.

\section{Test-retest Reliability}

To address test-retest reliability (Research Questions 1a and 4a), we used the elastic net models trained on Samples 1-3 to assess personality traits separately for the two interviews 
completed by Sample 4 participants at each time point. Then we calculated the correlation $\left(r_{t t}\right)$ between these two trait scores for each Big Five trait to index test-retest reliability, as reported in Table 7 and summarized here. Of the models developed on Sample 1, test-retest reliability for self-report models averaged $\bar{r}_{t t}=.36$ and ranged $.01-.69$, and for interviewer-report models averaged $\bar{r}_{t t}=.49$ and ranged $.37-.70$. Of the models developed on Sample 2, test-retest reliability for self-report models averaged $\bar{r}_{t t}=.51$ and ranged .16-.85, and for interviewer-report models averaged $\bar{r}_{t t}=.54$ and ranged .09-.76. Of the models developed on Sample 3, test-retest reliability for self-report models averaged $\bar{r}_{t t}=.50$ and ranged $.24-.85$, and for interviewer-report models averaged $\bar{r}_{t t}=.48$ and ranged $.23-.65$. On average, self- and interviewer-report models exhibited similar test-retest reliability $\left(\bar{r}_{t t \text { Self }}=.46 ; \bar{r}_{t t \text { Interviewer }}=.50\right)$. These test-retest correlations are, on average, higher than those observed in prior studies of interviews that occurred over a much longer duration (Schleicher et al., 2010), yet noticeably lower than selfreport personality scales.

Self-report models exhibited the lowest test-retest reliability for conscientiousness $\left(\bar{r}_{t t}=\right.$ $.30)$, openness $\left(\bar{r}_{t t}=.32\right)$, and extraversion $\left(\bar{r}_{t t}=.34\right)$, and highest for emotional stability $\left(\bar{r}_{t t}=\right.$ .76). On the other hand, interviewer-report models exhibited the lowest test-retest reliability for emotional stability $\left(\bar{r}_{t t}=.23\right)$ and highest for extraversion $\left(\bar{r}_{t t}=.70\right)$ and conscientiousness $\left(\bar{r}_{t t}=\right.$ $.65)^{4}$.

\section{Generalized Coefficient of Equivalence and Stability}

To address Research Questions $1 \mathrm{~b}$ and $4 \mathrm{~b}$, we used the same trait estimates described in

\footnotetext{
${ }^{4}$ Notably, an earlier version of this paper used only LIWC variables as verbal behavior predictors, finding higher test-retest reliability than the present investigation (Sample 1 self-report models $\bar{r}_{t t}=.63$, interviewer-report models $\bar{r}_{t t}=.56$; Sample 2 self-report models $\bar{r}_{t t}=.68$, interviewer-report models $\bar{r}_{t t}=.63$; Sample 3 self-report models $\bar{r}_{t t}=$ .51 , interviewer-report models $\bar{r}_{t t}=.56$ ). This likely occurs because $n$-grams are likely to vary more than the conceptual categories to which words belong, which is relevant to the fact that interviews provide interviewees with an open-ended prompt that may elicit vastly different responses depending on the occasion.
} 
Table 7

Test-retest reliability and generalized coefficient of equivalence and stability estimates for AVIPAs applied to Sample 4

$r_{t t} \quad \mathrm{GCES}_{\mathrm{s} 1 \mathrm{~s} 2} \quad \mathrm{GCES}_{\mathrm{s} 1 \mathrm{~s} 3} \quad \mathrm{GCES}_{\mathrm{s} 2 \mathrm{~s} 3}$ GCES

\begin{tabular}{|c|c|c|c|c|c|}
\hline \multicolumn{6}{|l|}{ Self-report models } \\
\hline \multicolumn{6}{|l|}{ Sample 1} \\
\hline Extraversion & .31 & .00 & -.08 & & .02 \\
\hline Agreeableness & .29 & .06 & -.15 & & .04 \\
\hline Conscientiousness & .51 & .08 & .09 & & .09 \\
\hline Emotional Stability & .69 & .14 & .28 & & .29 \\
\hline Openness & .01 & -.11 & -.06 & & -.03 \\
\hline \multicolumn{6}{|l|}{ Sample 2} \\
\hline Extraversion & .45 & .00 & & .15 & .02 \\
\hline Agreeableness & .85 & .06 & & .21 & .04 \\
\hline Conscientiousness & .16 & .08 & & .09 & .09 \\
\hline Emotional Stability & .75 & .14 & & .46 & .29 \\
\hline Openness & .34 & -.11 & & .06 & -.03 \\
\hline \multicolumn{6}{|l|}{ Sample 3} \\
\hline Extraversion & .26 & & -.08 & .15 & .02 \\
\hline Agreeableness & .55 & & -.15 & .21 & .04 \\
\hline Conscientiousness & .24 & & .09 & .09 & .09 \\
\hline Emotional Stability & .85 & & .28 & .46 & .29 \\
\hline \multirow[t]{2}{*}{ Openness } & .60 & & -.06 & .06 & -.03 \\
\hline & $r_{t t}$ & $\mathrm{GCES}_{\mathrm{s} 1 \mathrm{~s} 2}$ & $\mathrm{GCES}_{\mathrm{s} 1 \mathrm{~s} 3}$ & $\mathrm{GCES}_{\mathrm{s} 2 \mathrm{~s} 3}$ & GCES \\
\hline \multicolumn{6}{|c|}{ Interviewer-report models } \\
\hline \multicolumn{6}{|c|}{ Sample 1} \\
\hline Extraversion & .70 & .69 & .62 & & .66 \\
\hline Agreeableness & .45 & .21 & .13 & & .19 \\
\hline Conscientiousness & .54 & .50 & .43 & & .53 \\
\hline Emotional Stability & .37 & .07 & .01 & & .05 \\
\hline Openness & .40 & .17 & .26 & & .31 \\
\hline \multicolumn{6}{|l|}{ Sample 2} \\
\hline Extraversion & .74 & 69 & & .65 & .66 \\
\hline Agreeableness & 60 & .21 & & .25 & .19 \\
\hline Conscientiousness & .76 & .50 & & .66 & .53 \\
\hline Emotional Stability & .09 & .07 & & .03 & .05 \\
\hline Openness & .53 & .17 & & .50 & .31 \\
\hline \multicolumn{6}{|l|}{ Sample 3} \\
\hline Extraversion & .65 & & .62 & .65 & .66 \\
\hline Agreeableness & .24 & & .13 & .25 & .19 \\
\hline Conscientiousness & .65 & & .43 & .66 & .53 \\
\hline Emotional Stability & .23 & & .01 & .03 & .05 \\
\hline Openness & .62 & & .26 & .50 & .31 \\
\hline
\end{tabular}

Note: All statistics based on AVI-PA scores for the two interviews in Sample 4. $r_{t t}=$ test-retest reliability. GCES $_{\mathrm{s} 1 \mathrm{~s} 2}=$ GCES calculated using only the models from samples 1 and 2 . GCES $_{\mathrm{s} 1 \mathrm{~s} 3}=$ GCES calculated using only the models from samples 1 and 3 . GCES s $2 \mathrm{~s} 3_{3}=$ GCES calculated using only the models from samples 2 and 3. GCES = GCES calculated using all three samples' models. 
the test-retest reliability section but calculated cross-model test-retest correlations and averaged them together to derive the GCES, as reported in Table 7. The GCES is estimated by correlating two different measures of a construct administered at two different time points. Because AVIPAs can be applied to both time points, we calculated the average of such correlations for all three AVI-PAs (GCES), as well as for each pair of AVI-PAs $\left(\operatorname{GCES}_{\mathrm{s} 1 \mathrm{~s} 2}, \mathrm{GCES}_{\mathrm{s} 2 \mathrm{~s} 3}\right.$, and $\left.\mathrm{GCES}_{\mathrm{s} 2 \mathrm{~s} 3}\right)$. For example, $\mathrm{GCES}_{\mathrm{s} 1 \mathrm{~s} 2}$ was calculated by correlating Sample 1 models' trait scores of Sample 4 at Time 1 with the Sample 2 models' trait scores of Sample 4 at Time 2, correlating Sample 2 models' trait scores of Sample 4 at Time 1 with Sample 1 models' trait scores of Sample 4 at Time 2, then averaging these two correlations.

Because GCES estimates all sources of error (i.e., transient, random, and scale-specific) simultaneously and seeks to estimate a generalizable test-retest reliability statistic (across different AVI-PA models), GCES will typically be lower than test-retest correlations (Le et al., 2009). As can be seen in Table 7, the GCES for self-report models was extremely low. The strongest (yet still weak) evidence of reliability was for emotional stability assessments $\left(\mathrm{GCES}_{\mathrm{s} 2 \mathrm{~s} 3}=.46\right)$. Although there are not readily available benchmarks for comparing GCES, there is little evidence here to suggest that AVI-PAs trained on self-reports are reliable or that the different models score self-reports similarly.

The GCES for interviewer-report models was better for extraversion, conscientiousness, and openness, but not for agreeableness and emotional stability. Both the overall and paired GCES estimates for extraversion exceeded .60 . The paired Sample 2 and 3 GCES $_{\mathrm{s} 2 \mathrm{~s} 3}=.66$ for conscientiousness, while the overall conscientiousness GCES $=.53$. The $\mathrm{GCES}_{\mathrm{s} 2 \mathrm{~s} 3}=.50$ for openness, but the overall openness GCES $=.31$. None of the agreeableness GCES estimates exceeded .25 , and none of the emotional stability GCES estimates exceeded .07 . 
In response to Research Questions $1 \mathrm{~b}$ and $4 \mathrm{~b}$, there is promising evidence of reliability for the AVI-PAs trained on interviewer-reports for extraversion, conscientiousness, and to a lesser extent, openness. But for the remaining source-trait pairs, reliability is poor to absent.

\section{Convergent and Discriminant Relations Generalizability}

To investigate cross-sample convergent and discriminant relations (Research Questions 4c-4d), we used MTMM indices (Woehr et al., 2012) to summarize cross-sample trait assessments in Samples 1-3. First, we calculated the average convergence between reported traits and AVI-PAs (as reported in Appendix Table A2). For self-report models, C1 was .07, .09, and .07 . With regard to specific traits, the lowest cross-validated convergence was observed for conscientiousness $(\bar{r}=.04)$ and the highest convergence was observed for emotional stability $(\bar{r}$ $=.10$ ). Because convergence was so low and is a necessary condition to further explore validity (Woehr et al., 2012), we did not further investigate the generalizability of the validity evidence for self-report models.

For interviewer-report models, we again calculated the C1, D1, D2, and MV MTMM indices, as reported in Table 8. We calculated them for each set of assessments, then calculated their sample size weighted average. Appendix Tables A3-A5 report the MTMM matrices for the cross-sample assessments by the three sets of models (e.g., the top half of Table A3 contains correlations between Sample 1's AVI-PAs of Sample 2's participants and Sample 2's interviewer-reports, and the bottom half contains correlations between Sample 1's AVI-PAs of Sample 3's participants and Sample 3's interviewer-reports).

For these models, $\mathrm{C} 1$ averaged $M=.37$ and ranged from .34 (Sample 1) to .41 (Sample

2). D1 averaged $M=.17$ and ranged from .14 (Sample 3) to .20 (Sample 2), and D2 averaged $M$ $=.06$ and ranged from .02 (Sample 3) to .09 (Sample 2). MV averaged $M=.10$ and ranged from 
Table 8

Multitrait-multimethod statistics for AVI-PAs during cross-sample cross-validation

\begin{tabular}{lcccccc}
\hline & C1 & D1 & D2 & MV & D2 $2_{a}$ & MV $_{\mathrm{a}}$ \\
\hline Sample 1 Interviewer-report Models & .34 & .17 & .08 & .08 & .15 & .02 \\
Sample 2 Interviewer-report Models & .41 & .20 & .09 & .11 & .08 & .12 \\
Sample 3 Interviewer-report Models & .36 & .14 & .02 & .12 & -.03 & .17 \\
\hline
\end{tabular}

Note. $\mathrm{C} 1$ = convergence index. $\mathrm{D} 1=$ discrimination index $1 . \mathrm{D} 2=$ discrimination index $2 . \mathrm{MV}=$ method variance. $\mathrm{D} 2_{\mathrm{a}}=$ discrimination index 2 calculated using only automated video interviews' heterotrait-monomethod correlations. $\mathrm{MV}_{\mathrm{a}}=$ method variance for automated video interviews. Values are the sample size weighted average of the two sets of cross-sample assessments.

.08 (Sample 1) to .12 (Sample 3). D2 a averaged $M=.07$ and ranged from -.03 (Sample 3) to .15 (Sample 1), and $\mathrm{MV}_{\mathrm{a}}$ averaged $M=.10$ and ranged from .02 (Sample 1) to .17 (Sample 3).

Models trained on Sample 1 exhibited the largest drop in convergence $(\Delta \mathrm{C} 1=-.07)$, largely due to significant decreases in convergence for agreeableness $\left(r_{\text {within }}=.41\right.$ vs. $r_{\text {between }}=.28 ; z=2.22$, $p=.03)$ and emotional stability ( $r_{\text {within }}=.32$ vs. $\left.r_{\text {between }}=.18 ; z=2.25, p=.02\right)$. Convergence dropped only slightly for models trained on Sample $2(\Delta \mathrm{C} 1=-.01)$, with a significant decrease in convergence for agreeableness $\left(r_{\text {within }}=.44\right.$ vs. $\left.r_{\text {between }}=.28 ; z=2.96, p=.003\right)$ but a significant increase in convergence for conscientiousness ( $r_{\text {within }}=.41$ vs. $r_{\text {between }}=.52 ; z=2.25, p=.02$ ). Convergence dropped slightly more for models trained on Sample $3(\Delta \mathrm{C} 1=-.02)$, primarily due to a significant decrease in convergence for openness $\left(r_{\text {within }}=.41\right.$ vs. $r_{\text {between }}=.27 ; z=2.38, p=$ .02). The interviewer-report models from Samples 2 and 3 had consistent MTMM indices when calculated within- and between-samples, although the models from Sample 1 still exhibited better discriminant evidence and less method variance compared to the models from Sample 3. In line with the within-sample investigations, the convergent evidence of validity was strongest for extraversion and second strongest for conscientiousness. Specifically, the convergence of the AVI extraversion assessments again exceeded $\operatorname{ICC}(1,1)(\bar{r}=.64)$, and Sample 2's AVI conscientiousness assessment exceeded ICC $(1,1)(r=.52)$, while Sample 3's AVI 
conscientiousness assessment was very similar to $\operatorname{ICC}(1,1)(r=.45)$.

\section{Test Content: Relative Contribution of Verbal, Paraverbal, and Nonverbal Behavior and}

\section{Common Predictors}

To address Research Question 5a, we explored the relative contribution of each type of behavior to the final interviewer-report elastic net regression models. To do so, we trained models on a version of the data with all variables standardized by subtracting their within-sample mean and dividing by their standard deviation. Then, we summed the regression weights from each type of behavior (i.e., verbal, paraverbal, and nonverbal) within each model and divided that value by the overall sum of regression weights in that model. The proportion of regression weights accounted for by each type of behavior is summarized in Figure 3. To answer Research Question 6b, we checked whether same-trait interviewer-report models exhibit overlap across samples in terms of the predictors selected by elastic net regression.

Figure 3

Relative contribution of each type of behavior in interviewer-report models

\section{a. Sample 1}
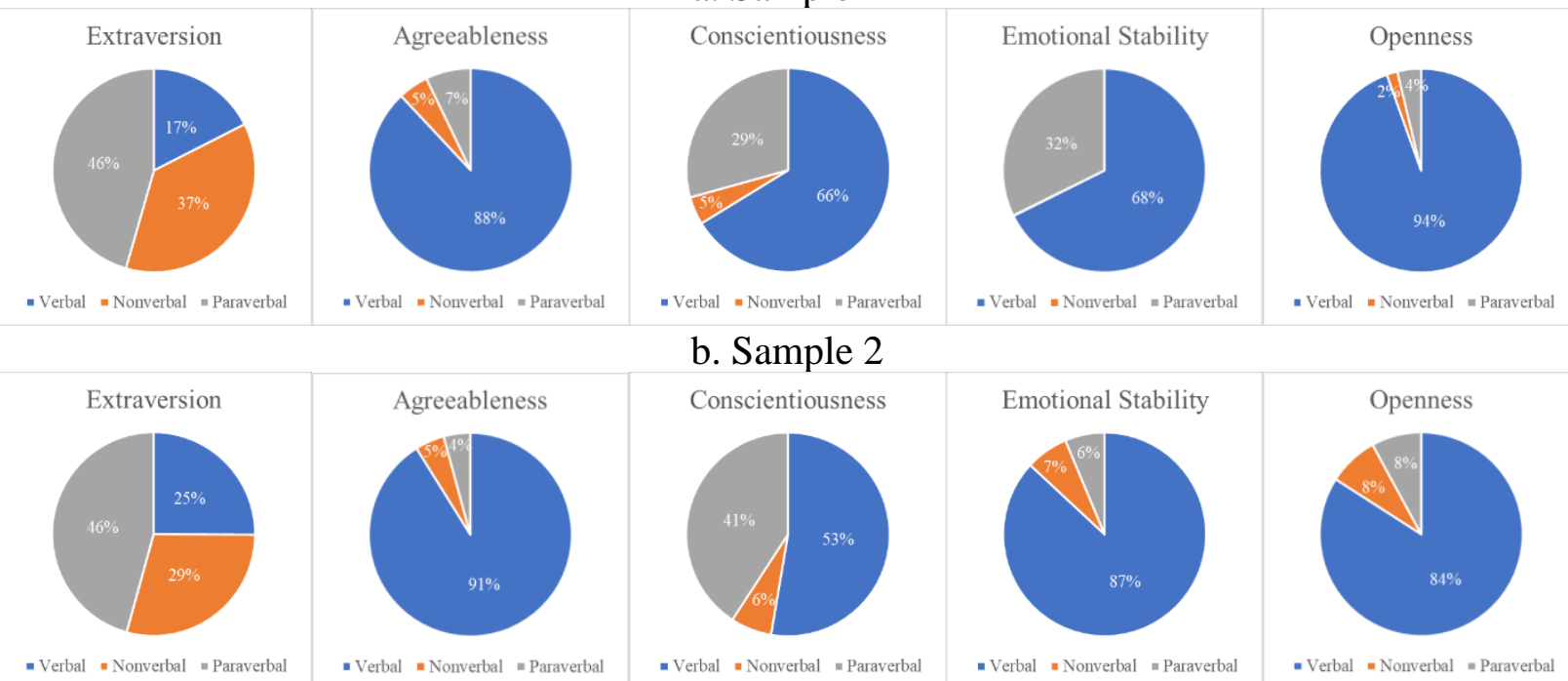

\section{b. Sample 2}
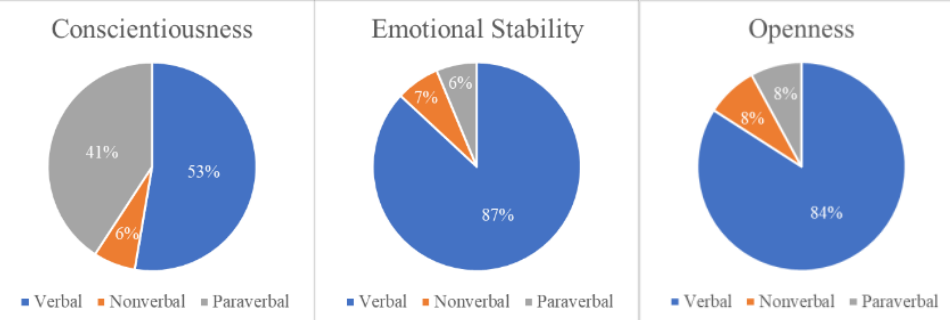

c. Sample 3 

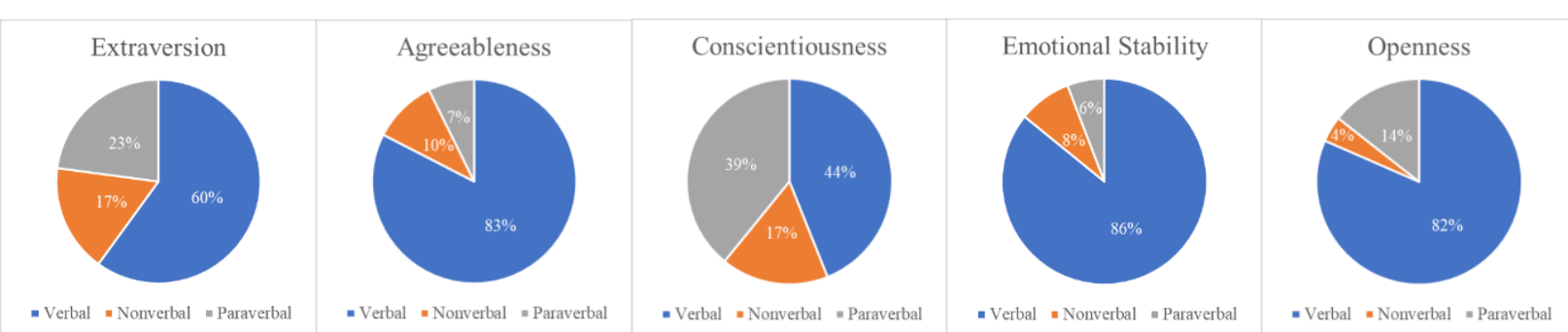

Note: Relative contribution calculated by summing all standardized regression weights in the final model from each type of behavior, then dividing that sum by the sum of the standardized regression weights from all three types of behavior in the model. Percentages represent the percentage of standardized regression weights given to predictors of that type.

As shown in Figure 3, there were relatively consistent differences between traits in the relative contribution of each type of behavior. On average, extraversion models weighted paraverbal behavior highest (38\%), verbal behavior second highest (34\%), and nonverbal behavior lowest (28\%). Only extraversion models did not weight verbal behavior highest of the three types of behavior. Interestingly, although extraversion models exhibited the highest crossvalidated convergent correlations, they also exhibited the most within-trait differences in relative contributions, as verbal behavior regression weights comprised 17.5\% of all weights in Sample 1 but $60.0 \%$ of all weights in Sample $3(\mathrm{SD}=22.7 \%)$. In other words, extraversion models had the highest variance in terms of the relative contribution of each type of behavior. Four behavioral cues were positive predictors of extraversion in all three interviewer-report models: word count, average loudness (i.e., volume), average loudness peaks per second (i.e., speech rate), and the standard deviation of facial AU12 (zygomatic major; the mouth part of a smile) activation intensity. No other set of same-trait models had more than three common predictors.

On average, conscientiousness models utilized verbal behavior for over half of the total regression weights (54.3\%). Nonverbal behavior comprised just $9.3 \%$ of the regression weights, and paraverbal behavior comprised $36.4 \%$ of the regression weights. In all three samples, the final models included word count and proportion of words longer than six letters as positive 
predictors, and the assent (e.g., agree, OK, yes) category from LIWC as a negative predictor.

The remaining models, on average, utilized verbal behavior for over four-fifths of the regression weights $(87.2 \%, 80.1 \%$, and $86.7 \%$ respectively for agreeableness, emotional stability, and openness models). As regards Research Question 6a, models tended to be dominated by verbal behavior, except for extraversion models. For these remaining models, some meaningful predictors emerged across multiple samples. For agreeableness models, the $n$ gram love and average harmonics to noise ratio (i.e., lack of hoarseness) were positive predictors in all three samples' models. Additionally, stemmed $n$-grams containing help were positive predictors in all three agreeableness models (help in Sample 1; help, abl help, help peopl, peopl help, help like, way help, help need, and help one in Sample 2; help just, help well, help peopl, and go help in Sample 3). For emotional stability models, the cooccurrence of facial AU 20 (risorius; lip stretcher) and AU 25 (depressor labii, relaxation of mentalis, and orbicularis oris; lips part) was a negative predictor in all three samples' models, albeit with low weight. Facial AU 20 is associated with fear (Ekman \& Friesen, 1978), and cooccurrence with AU 25 suggests some negative emotions or tension while speaking. Additionally, the anxiety and negative emotion LIWC categories were negative predictors of emotional stability in two of the three models.

For openness models, $n$-grams containing one and love were positive predictors in all three samples' models (thing one and realli love in Sample 1; work one and realli love in Sample 2; one first, one, and love in Sample 3). Further, the stemmed $n$-gram creativ was positively predictive of openness and was weighted highest of all predictors in Sample 1's openness model and second highest in Sample 2's openness model. As regards Research Question 6b, common predictors were found in same-trait models for all five traits. Overall, these common predictors 
appeared intuitively to be conceptually relevant to their respective focal trait.

\section{Nomological Network}

Regarding Research Question 6, we examined bivariate correlations and used multiple regression to examine how AVI-PAs related to academic outcomes in Samples 2 and 3. Table 9 reports the bivariate correlations between AVI-PA scores and academic outcomes for models trained on interviewer-reports in Samples 1 and 3, assessing Sample 2 participants' traits, and Table 10 reports the results of hierarchical regression. Tables 11 and 12 report the same results for models trained on interviewer-reports in Samples 1 and 2, assessing Sample 3 participants' traits. In the regression results, Model 1 is multiple regression with one set of AVI-PA scores as predictors (e.g., either Sample 1 or Sample 3's AVI scores of Sample 2 participants' traits were entered as predictors). Model 2 is multiple regression with self- and interviewer-reported traits as predictors (only reports $\mathrm{R}^{2}$ for simplicity of presentation), and Model 3 tests whether AVI-PAs increment beyond self- and interviewer-reported traits in predicting these outcomes.

Table 9

Bivariate correlations between Sample 2 academic outcomes and cross-sample AVI-PA scores (interviewer-report models)

\begin{tabular}{lcllll}
\hline & $\begin{array}{c}\text { College } \\
\text { GPA }\end{array}$ & $\begin{array}{c}\text { HS } \\
\text { GPA }\end{array}$ & $\begin{array}{c}\text { SAT } \\
\text { Verbal }\end{array}$ & $\begin{array}{c}\text { SAT } \\
\text { Math }\end{array}$ & ACT \\
\hline Sample 1 AVI & & & & & \\
Extraversion & -.04 & $.22^{* *}$ & .02 & $-.14^{*}$ & $.18^{*}$ \\
Agreeableness & .06 & $.14^{*}$ & -.07 & $-.24^{* *}$ & -.09 \\
Conscientiousness & .11 & $.11^{*}$ & $.10^{\dagger}$ & .00 & .09 \\
Emotional Stability & .02 & .07 & .07 & .07 & $.15^{*}$ \\
Openness & -.03 & .07 & -.07 & -.09 & .08 \\
Sample 3 AVI & & & & & \\
Extraversion & -.01 & $.21^{* *}$ & -.03 & $-.18^{* *}$ & $.12^{\dagger}$ \\
Agreeableness & .02 & $.12^{*}$ & $-.10^{\dagger}$ & $-.22^{* *}$ & -.06 \\
Conscientiousness & .04 & $.21^{* *}$ & $.11^{\dagger}$ & .02 & $.20^{* *}$ \\
Emotional Stability & .00 & $.09^{\dagger}$ & -.04 & $.10^{\dagger}$ & .11 \\
Openness & .04 & $.16^{* *}$ & .05 & -.05 & $.15^{*}$ \\
\hline
\end{tabular}

Note: ${ }^{\dagger} p<.1 . * p<.05 . * * p<.01$ 
Sample 2. Table 9 reports the bivariate correlations for participants in Sample 2. In terms of specific trait-outcome effect sizes, Bosco et al. (2015) suggest that correlations between psychological characteristics, like personality, and performance outcomes are medium in size when $r>.10$ and $<.23$. Here we summarize all effects at least medium in size. Sample 1 model's predictions of conscientiousness correlated with Sample 2 college GPA $r=.11$. Sample 1 models' predictions of extraversion, agreeableness, and conscientiousness correlated with Sample 2 high school GPA $r \mathrm{~s}=.22, .14$, and .11 respectively, and Sample 3 models' predictions of extraversion, agreeableness, conscientiousness, and openness correlated with Sample 2 high school GPA $r s=.21, .12, .21$, and .16 . Sample 3 model's predictions of conscientiousness correlated with Sample 2 SAT verbal scores $r=.11$. Sample 1 and 3 models' predictions of extraversion and agreeableness were correlated with SAT math $r$ s $=-.14$ and -.24 (Sample 1 models) and $r$ s $=-.18$ and -.22 (Sample 3 models). Sample 1 models' predictions of extraversion and emotional stability correlated with ACT scores $r \mathrm{~s}=.18$ and .15 , and Sample 3 models' predictions of extraversion, conscientiousness, emotional stability, and openness correlated with ACT scores $r s=.12, .20, .11$, and .15 , respectively.

Table 10 reports the regression results for Sample 2. The interviewer-report model assessments from Samples 1 and 3 were significant predictors of high school GPA, SAT math, and ACT scores, and trait estimates derived from Sample 3 models were also significant predictors of SAT verbal scores $\left(\mathrm{R}^{2}=.06\right)$. Both sets of model assessments incrementally predicted high school GPA and SAT math scores beyond reported traits $\left(\Delta \mathrm{R}^{2}=.02 \& .06\right.$ for Sample 1 models; $\Delta \mathrm{R}^{2}=.03 \& .07$ for Sample 3 models). For all outcomes except college GPA, Sample 3 models' predictions explained at least half as much variance (in absolute terms) as both self- and interviewer-reports. 
Table 10

Regression estimates predicting academic outcomes: Sample 2 cross-sample AVI-PA scores (interviewer-report models)

\begin{tabular}{|c|c|c|c|c|c|c|c|c|c|c|}
\hline & \multicolumn{2}{|c|}{$\begin{array}{l}\text { College GPA } \\
(\mathrm{N}=161)\end{array}$} & \multicolumn{2}{|c|}{$\begin{array}{l}\text { HS GPA } \\
(\mathrm{N}=375)\end{array}$} & \multicolumn{2}{|c|}{$\begin{array}{l}\text { SAT Verbal } \\
(\mathrm{N}=302)\end{array}$} & \multicolumn{2}{|c|}{$\begin{array}{l}\text { SAT Math } \\
(\mathrm{N}=306)\end{array}$} & \multicolumn{2}{|c|}{$\begin{array}{c}\text { ACT } \\
(\mathrm{N}=222)\end{array}$} \\
\hline & M 1 & M $2 / 3$ & M 1 & M $2 / 3$ & M 1 & M 2/3 & M 1 & M $2 / 3$ & M 1 & M $2 / 3$ \\
\hline \multicolumn{11}{|l|}{ Self-reports } \\
\hline \multicolumn{11}{|l|}{ Interviewer-reports } \\
\hline$R^{2}$ & & $.11^{\dagger}$ & & $.12 * *$ & & $.10^{* *}$ & & $.18^{* *}$ & & $.17 * *$ \\
\hline \multicolumn{11}{|l|}{ Sample 1 AVI } \\
\hline Extraversion & -.07 & -.16 & $.19^{* *}$ & $.17 *$ & .02 & -.05 & $-.12^{\dagger}$ & $-.19 * *$ & $.17^{*}$ & .12 \\
\hline Agreeableness & .07 & .09 & .08 & .05 & $-.08^{*}$ & -.07 & $-.22 * *$ & $-.16 * *$ & $-.15^{*}$ & $-.13^{\dagger}$ \\
\hline Conscientiousness & .11 & .08 & .07 & .04 & .09 & .04 & .01 & -.08 & .04 & .02 \\
\hline Emotional Stability & .01 & -.02 & .01 & .02 & .05 & .03 & $.11^{\dagger}$ & .06 & .10 & .09 \\
\hline Openness & -.03 & .00 & .03 & .01 & -.08 & $-.10^{\dagger}$ & -.06 & -.05 & .06 & .06 \\
\hline$R^{2}$ & .02 & .13 & $.06^{* *}$ & $.14 * *$ & .02 & $.12 * *$ & $.08 * *$ & $.24 * *$ & $.07 * *$ & $.20 * *$ \\
\hline$\Delta R^{2}$ & & .02 & & $.02 * *$ & & .02 & & $.06^{* *}$ & & .03 \\
\hline \multicolumn{11}{|l|}{ Sample 3 AVI } \\
\hline Extraversion & -.09 & -.09 & $.14^{\dagger}$ & .09 & $-.17 *$ & $-.20 *$ & $-.30 * *$ & $-.28 * *$ & -.00 & -.05 \\
\hline Agreeableness & .01 & .03 & .02 & .04 & $-.15^{*}$ & -.09 & $-.24 * *$ & $-.16 * *$ & $-.19 * *$ & $-.14 *$ \\
\hline Conscientiousness & .06 & .02 & .11 & .12 & $.26 * *$ & $.18 *$ & $.19^{*}$ & .05 & $.20 *$ & $.17^{\dagger}$ \\
\hline Emotional Stability & .01 & .02 & .03 & .03 & -.09 & $-.10^{\dagger}$ & $.10^{\dagger}$ & .06 & .05 & .04 \\
\hline Openness & .07 & .07 & .00 & .00 & .10 & .06 & $.13^{\dagger}$ & .06 & .13 & .11 \\
\hline$R^{2}$ & .01 & .12 & $.06^{* *}$ & $.15^{* *}$ & $.06^{* *}$ & $.13^{* *}$ & $.11^{* *}$ & $.25^{* *}$ & $.08 * *$ & $.21 * *$ \\
\hline$\Delta R^{2}$ & & .01 & & $.03 *$ & & $.03^{\dagger}$ & & $.07 * *$ & & .04 \\
\hline
\end{tabular}

Note: ${ }^{\dagger} p<.1 . * p<.05 . * * p<.01$. Table entries are standardized regression coefficients. AVI $=$ automated video interviews. M $1=$ model 1 , using one set of interviewer-report AVI-PAs only as predictors. M 2/3 = models 2 and 3. Model 2 uses self- and interviewer-reported traits only, and Model 3 adds one set of AVI-PAs.

Sample 3. Table 11 reports the bivariate correlations for participants in Sample 3. In terms of specific trait-outcome effect sizes, Sample 1 models' predictions of extraversion, agreeableness, and openness correlated with Sample 3 high school GPA $r s=.12, .11$, and .13, and Sample 2 models' predictions of extraversion and conscientiousness correlated with high school GPA $r s=.13$ and .11. Sample 1 models' predictions of extraversion and conscientiousness correlated with SAT verbal $r \mathrm{~s}=.12$ and .19 , and Sample 2 models' predictions of agreeableness and conscientiousness correlated with SAT verbal $r$ s $=-.17$ and .19 . Sample 1 
and 2 models' predictions of agreeableness correlated with SAT math $r \mathrm{~s}=-.12$ and -.25 , respectively. Sample 1 models' predictions of extraversion, conscientiousness, and openness correlated with ACT scores $r \mathrm{~s}=.12, .17$, and .12 , and Sample 2 models' predictions of agreeableness, conscientiousness, emotional stability, and openness correlated with ACT scores $r \mathrm{~s}=-.18, .22, .12$, and .16 .

Table 11

Bivariate correlations between Sample 3 academic outcomes and cross-sample AVI-PA scores (interviewer-report models)

\begin{tabular}{lccccc}
\hline & $\begin{array}{c}\text { College } \\
\text { GPA }\end{array}$ & $\begin{array}{c}\text { HS } \\
\text { GPA }\end{array}$ & $\begin{array}{c}\text { SAT } \\
\text { Verbal }\end{array}$ & $\begin{array}{c}\text { SAT } \\
\text { Math }\end{array}$ & ACT \\
\hline Sample 1 AVI & & & & & \\
Extraversion & .01 & $.12^{*}$ & $.12^{\dagger}$ & -.02 & .12 \\
Agreeableness & .09 & $.11^{\dagger}$ & .01 & $-.12^{\dagger}$ & .09 \\
Conscientiousness & -.05 & .09 & $.19^{* *}$ & -.05 & $.17^{*}$ \\
Emotional Stability & -.03 & -.07 & -.08 & -.02 & .01 \\
Openness & .00 & $.13^{*}$ & .10 & -.05 & .12 \\
Sample 2 AVI & & & & & \\
Extraversion & .00 & $.13^{*}$ & .11 & -.03 & .10 \\
Agreeableness & .06 & $.10^{\dagger}$ & $-.17^{*}$ & $-.25^{* *}$ & $-.18^{*}$ \\
Conscientiousness & .05 & $.11^{\dagger}$ & $.19^{*}$ & .00 & $.22^{* *}$ \\
Emotional Stability & .05 & .07 & .10 & -.09 & .12 \\
Openness & -.02 & .07 & .10 & .07 & $.16^{*}$ \\
\hline Note: ${ }^{\dagger} p<.1{ }^{*} p<.05 .{ }^{* *} p<.01$ & & & &
\end{tabular}

Table 12 reports the regression results for Sample 3. Sample 1's interviewer-report model assessments were marginally significant predictors of high school GPA and SAT verbal scores and provided a marginally significant incremental prediction of high school GPA beyond reported traits $\left(\Delta \mathrm{R}^{2}=.03\right)$. Sample 2's interviewer-report model assessments were significant predictors of SAT verbal, SAT math, and ACT scores and provided a marginally significant incremental prediction of SAT math scores $\left(\Delta \mathrm{R}^{2}=.04\right)$. Sample 2 models' predictions explained at least half as much variance as reported traits in SAT verbal and SAT math scores, and a little less than half as much for ACT scores. 
Table 12

Regression estimates predicting academic outcomes: Sample 3 cross-sample AVI-PA scores (interviewer-report models)

\begin{tabular}{|c|c|c|c|c|c|c|c|c|c|c|}
\hline & \multicolumn{2}{|c|}{$\begin{array}{l}\text { College GPA } \\
(\mathrm{N}=270)\end{array}$} & \multicolumn{2}{|c|}{$\begin{array}{l}\text { HS GPA } \\
(\mathrm{N}=282)\end{array}$} & \multicolumn{2}{|c|}{$\begin{array}{l}\text { SAT Verbal } \\
(\mathrm{N}=225)\end{array}$} & \multicolumn{2}{|c|}{$\begin{array}{c}\text { SAT Math } \\
(\mathrm{N}=226)\end{array}$} & \multicolumn{2}{|c|}{$\begin{array}{c}\text { ACT } \\
(\mathrm{N}=162)\end{array}$} \\
\hline & M 1 & M $2 / 3$ & M 1 & M 2/3 & M 1 & M $2 / 3$ & M 1 & M $2 / 3$ & M 1 & M $2 / 3$ \\
\hline \multicolumn{11}{|l|}{ Self-reports } \\
\hline \multicolumn{11}{|l|}{ Interviewer-reports } \\
\hline$R^{2}$ & & $.12^{* *}$ & & $.09^{*}$ & & $.14^{* *}$ & & $.11 *$ & & $.19 * *$ \\
\hline \multicolumn{11}{|l|}{ Sample 1 AVI } \\
\hline Extraversion & .02 & -.06 & .07 & .13 & .08 & .05 & .02 & -.00 & .09 & .01 \\
\hline Agreeableness & .09 & .03 & .08 & .05 & -.03 & .00 & $-.12^{\dagger}$ & -.08 & .05 & .07 \\
\hline Conscientiousness & -.09 & $-.15^{*}$ & .02 & .04 & $.16^{*}$ & .10 & -.06 & -.09 & $.19^{*}$ & .12 \\
\hline Emotional Stability & .05 & .03 & .02 & -.00 & -.00 & .00 & -.05 & -.07 & .14 & $.15^{\dagger}$ \\
\hline Openness & .00 & -.03 & .10 & $.12 *$ & .05 & .05 & -.03 & -.03 & .06 & .04 \\
\hline$R^{2}$ & .01 & $.14 * *$ & $.03^{\dagger}$ & $.12^{* *}$ & $.05^{\dagger}$ & $.15^{* *}$ & .01 & $.12 *$ & .06 & $.21 * *$ \\
\hline$\Delta R^{2}$ & & .02 & & $.03^{\dagger}$ & & .01 & & .01 & & .02 \\
\hline \multicolumn{11}{|l|}{ Sample 2 AVI } \\
\hline Extraversion & -.03 & -.00 & .10 & $.18^{\dagger}$ & .02 & .04 & -.06 & -.03 & -.07 & -.02 \\
\hline Agreeableness & .05 & -.03 & .09 & .07 & $-.20 * *$ & $-.14 *$ & $-.24 * *$ & $-.17 *$ & $-.16^{*}$ & -.07 \\
\hline Conscientiousness & .08 & -.08 & .05 & .01 & $.20 * *$ & .13 & .06 & .03 & $.22 *$ & -.10 \\
\hline Emotional Stability & .02 & .05 & -.00 & .03 & .02 & -.04 & -.09 & -.12 & .04 & .21 \\
\hline Openness & -.05 & -.13 & -.01 & -.02 & -.01 & -.03 & .10 & .04 & .05 & -.05 \\
\hline$R^{2}$ & .01 & $.14 * *$ & .03 & $.11^{* *}$ & $.07 * *$ & $.16^{* *}$ & $.08^{* *}$ & $.14 * *$ & $.08 *$ & $.21 * *$ \\
\hline$\Delta R^{2}$ & & .02 & & .02 & & .02 & & $.04^{\dagger}$ & & .02 \\
\hline
\end{tabular}

Note: ${ }^{\dagger} p<.1 . * p<.05 . * * p<.01$. Table entries are standardized regression coefficients. AVI $=$ automated video interviews. M $1=$ model 1 , using one set of interviewer-report AVI-PAs only as predictors. M 2/3 = models 2 and 3. Model 2 uses self- and interviewer-reported traits only, and Model 3 adds one set of AVI-PAs.

\section{Discussion}

Automated video interviews are increasingly being adopted by organizations for earlystage applicant screening due to their potential to decrease costs and improve the quality of applicant screening. However, little evidence has been available to suggest that AVIs are psychometrically valid. This research's objective was to critically examine the psychometric properties of AVI-PAs as an example of one set of constructs that could be assessed with AVIs.

The first part of these investigations (i.e., within-sample) assessed the convergent and 
discriminant evidence of validity for AVI-PAs when tested with nested $k$-fold cross-validation. Among the models trained on self-reports, convergent evidence was minimal, with the most optimistic evidence for the less visible trait of emotional stability in Sample 1, emotional stability and openness in Sample 3, and the more visible traits of extraversion and agreeableness in Sample 2. By comparison, all three sets of interviewer-report models exhibited superior convergence. Regarding discriminant evidence for the AVI-PAs trained on self-reports, only those from Sample 3 had positive MTMM discrimination indices. These indices are positive when construct variance (i.e., monotrait-hetermethod correlations; C1) exceeds higherorder/common factor variance (i.e., heterotrait-heteromethod correlations; D1) and method variance (i.e., heterotrait-monomethod correlations; D2). All interviewer-report models had positive discrimination indices, and those from Samples 1 and 2 had larger discrimination indices than Sample 3's self-report models.

The second part of these investigations (i.e., cross-sample) examined the generalizability of the psychometric properties of AVI-PAs by applying the trained machine learning models to new interviews. We applied the thirty predictive models (trained separately on the self- and interviewer-reported Big Five traits from Samples 1, 2, and 3) to assess traits in a sample of participants (Sample 4) who completed a video interview twice to examine reliability. Overall, self-report models exhibited poor test-retest reliability for all traits except emotional stability $\left(\bar{r}_{\mathrm{tt}}\right.$ $=.76$ ), and GCES was low for all traits except emotional stability models from Samples 2 and 3 $\left(\mathrm{GCES}_{\mathrm{s} 2 \mathrm{~s} 3}=.46\right)$. Interviewer-report models exhibited evidence of test-retest reliability for extraversion $\left(\bar{r}_{\mathrm{tt}}=.70\right)$, conscientiousness $\left(\bar{r}_{\mathrm{tt}}=.65\right)$, and, to a lesser extent, openness $\left(\bar{r}_{\mathrm{tt}}=.52\right)$, but little evidence of test-retest reliability for agreeableness $\left(\bar{r}_{\mathrm{tt}}=.43\right)$ and emotional stability $\left(\bar{r}_{\mathrm{tt} .}=23\right)$. GCES was high for interviewer-report models of extraversion (GCES = .66), 
conscientiousness models from Samples 2 and $3\left(\operatorname{GCES}_{\mathrm{s} 2 \mathrm{~s} 3}=.66\right)$, and to a lesser extent, openness models from Samples 2 and $3\left(\mathrm{GCES}_{\mathrm{s} 2 \mathrm{~s} 3}=.50\right)$.

Regarding convergent relations, models trained on self-reports exhibited virtually no convergence with self-reported traits when applied to new samples $(\bar{r}=.07)$, whereas models trained on interviewer-reports exhibited cross-sample convergence similar to, but slightly lower than, when they were tested using nested $k$-fold cross-validation $(\bar{r}=.37)$. Because convergence is a necessary condition for further investigations of validity, we only investigated the generalizability of discriminant evidence for interviewer-report models. All interviewer-report models again demonstrated positive MTMM discrimination indices, although scores from Sample 3's models did not when isolating the properties of the AVI-PAs (i.e., $\mathrm{D} 22_{\mathrm{a}}$ and $\mathrm{MV}_{\mathrm{a}}$ ). Regarding the content of the AVI-PAs, the verbal, paraverbal, and nonverbal predictors across the models for each trait appeared to be conceptually relevant to the focal trait, and with the exception of extraversion, interviewer-report models used verbal behavior more than paraverbal or nonverbal behavior. However, there were inconsistencies in the relative contribution of verbal behavior in extraversion models, as the weight of verbal behavior in interviewer-report extraversion models changed by about $40 \%$ between Samples 1 and 3 . This suggests that, while the models trained on interviewer-reports may consistently predict interviewer-reported extraversion, the degree to which relevant verbal behaviors are used to make such ratings vary. Such inconsistency may be due to differences in interview questions, yet this did not appear to affect the convergence of the models. The interviewer-report model assessments predicted multiple academic outcomes beyond self- and interviewer-reported traits in Samples 2 and 3, and numerous medium-sized effects (Bosco et al., 2015) were observed between AVI-PAs and academic outcomes. Together, these sources of evidence lend initial 
support to interpreting AVI-PAs as substantively tapping into personality constructs.

\section{Theoretical Implications}

While some of the methods used in the present study are novel to many applied psychologists, AVIs are simply quantifying and selecting behaviors extracted from video clips and then weighting them as predictors in statistical models. This is an empirically-keyed assessment, in that machine learning algorithms select and weight behaviors to maximize the prediction of (i.e., convergence with) human reported traits, without regard for other psychometric properties. Therefore, for all AVIs, it is important to recognize the mediating processes that affect their reliability and validity. Figure 1 provides an initial conceptual framework that draws on prior personality (Connelly \& Ones, 2010; Funder, 1995) and employment interview (Huffcutt et al., 2011) research to identify some of these processes.

Drawing on Funder's RAM (1995) and its emphasis on the trait relevance and availability of behaviors, as well as the ability of observers to detect and utilize the behaviors to make trait judgments, we can understand the theoretical implications of our findings by characterizing AVIs as a special kind of rater that assesses personality by using behaviors to replicate human ratings. AVI-PAs will be more accurate to the extent that 1) personality-relevant behaviors are available and vary across participants and 2) the computer is able to detect personality-relevant behaviors. For example, the source of personality information (i.e., interviewer versus interviewee) appeared to affect the availability of personality-relevant behaviors by affecting which and how many behaviors were personality-relevant. Interviewees self-reported their context-independent traits, whereas interviewers gleaned an interview context-specific view of personality. Similar to how contextualized personality self-reports can improve criterion prediction (Shaffer \& Postlethwaite, 2012; Woo et al., 2015), the greater convergence of 
interviewer-reports suggests the importance of contextualized personality for understanding context-specific behaviors.

Situational characteristics can moderate the psychometric properties of AVI-PAs to the extent that they affect the relationship between personality and behavior. Situational characteristics in our study that may have had such effects include question consistency and question trait relevance. Question consistency is an aspect of interview structure (Chapman \& Zweig, 2005), and Sample 1 used relatively inconsistent questions as each group of respondents was encouraged to respond to one or more questions. In contrast, Sample 2 and 3's mock interviews were more consistent since all respondents within each sample answered the same prompts. Question trait relevance regards whether questions elicit behavioral expressions of specific traits (Tett \& Burnett, 2003; Tett \& Guterman, 2000). Only the questions in Sample 3 were designed to be directly relevant to the Big Five traits.

Because other elements changed between samples that may also affect the psychometric properties of AVI-PAs (e.g., sample size, number of questions), we can only offer tentative interpretations of these effects. For example, there were differences in convergent evidence during our within-sample investigations across traits for the self-report models. Emotional stability was relatively accurately assessed in Samples 1 and 3, extraversion and agreeableness were accurately assessed in Sample 2, and openness was accurately assessed in Sample 3. However, these effects were less evident for interviewer-reports as within-sample convergence was much more consistent across samples for those models. The cross-sample investigations suggest that the relationship between self-reported personality and interview performance (i.e., behavior) may not be consistent across situations. In contrast, the relationship between interviewer-reported traits and interview performance appears to be relatively more consistent. 
Such, cross-sample consistency may actually reflect trait-like perceiver effects on rating targets, shared stereotypes based on physical appearance, and common schema for interpreting behavioral cues (Kenny, 1991, 2004; Wood et al., 2010). These and other rater "errors" could actually reflect true construct variance (e.g., halo effects reflecting the accurate perception of the covariation of socially desirable qualities; Funder, 1995; Funder \& West, 1993).

\section{Practical Implications}

Automating video interviews hold the potential to save organizations time and money, and the present study provided initial evidence regarding AVI-PAs' reliability, convergent relations, discriminant relations, test content, and relationships with academic outcomes. Assessment at scale can bring considerable long-term benefits, even if slightly less valid than other approaches (Chamorro-Premuzic et al., 2017). Our study provides some initial evidence suggesting that AVI-PAs may validly assess some traits, but the evidence is mixed and many questions remain unanswered.

One promising area of evidence for AVI-PAs was construct discrimination. Practically, construct discrimination is a challenge in employment interviews (Hamdani et al., 2014). Yet, evidence of construct discrimination was much greater for the AVI-PAs than the facet-level personality interviews investigated by Van Iddekinge et al. (2005). Analyzing AVI models may help with identifying specific behaviors relevant to one KSAO but not another, and such insights could be used in the future to enhance interviewer frame-of-reference training.

The promising construct discrimination evidence was specific to AVI-PAs trained on interviewer-reports, making it clear that development choices affect the psychometric properties of AVI-PAs. If the goal of using AVI-PAs is to overcome issues with self-reported personality in selection, then interviewer-reports should likely be used. Encouragingly, compared to models 
trained on self-reports, models trained on interviewer-reports exhibited much stronger evidence of construct validity and generalized to new interview questions.

Another consideration is whether AVI vendors should allow clients to tailor interview questions for specific roles. Our results suggest that the psychometric properties of AVI-PAs may remain relatively consistent when models trained on one set of questions are used to assess interviewees who were asked a different set of questions. Samples 1 and 3's AVI-PA interviewer-report models exhibited worsened psychometric properties when used to assess cross-sample personality. Yet, the psychometric properties of Sample 2's AVI-PAs decreased only minimally when applied cross-sample $(\Delta \mathrm{C} 1=-.01, \Delta \mathrm{D} 1=-.03$, and $\Delta \mathrm{D} 2=-.01)$ and, in fact, exhibited higher convergence for conscientiousness $(\Delta r=.11)$ and less method variance $(\Delta \mathrm{MV}=-.02)$ compared to the within-sample investigations. Standardizing questions in AVIs may improve their psychometric properties, but the present study suggests that allowing clients to use interview questions different from those used to train the AVI-PAs may be justifiable.

However, more pieces of evidence are needed to justify the use of AVIs in personnel selection. Evidence based on response processes (AERA et al., 2014) could shed light on the ability of AVIs to discriminate between good and poor interview performance. This is also related to the content of AVIs - the behavioral predictors common to interviewer-report models for a given trait appeared intuitively related to the focal traits. For example, the extraversion models included behaviors related to talkativeness (i.e., word count, speech rate) and social energy (i.e., volume, mouth smiles). Future research should specify trait-relevant behaviors a priori and explore response processes to generate more robust content evidence of validity.

Second, although AVI-PAs can serve as an alternative to fakeable self-reports, it is not known whether AVI-PAs can be faked. Interviews appear to be less fakeable than self-reports 
(e.g., Van Iddekinge et al., 2005). However, this may not hold for AVI-PAs. Future studies that include faking or applicant-like conditions, as well as adversarial examples_-inputs meant to fool trained machine learning models into making mistakes (e.g., Goodfellow et al., 2014)—may help determine the extent to which faking affects the psychometric properties of AVIs.

Third, several vendors of AVIs promote their products as being fairer and less biased than traditional assessments (Raghavan et al., 2019). However, little evidence is generally made available beyond mean-level comparisons across legally protected groups, even though the Principles and Standards reject the equal outcomes definition of fairness (AERA et al., 2014; SIOP, 2018). Initial evidence suggests that automated interviews are associated with lower applicant reactions and perceived as less fair than traditional interviews (Langer et al., 2019). Additionally, algorithmic bias continues to be a widespread concern, and AVIs may be biased against Blacks and African Americans due to their use of facial recognition software that measures nonverbal behavior (EPIC, 2019; Harris et al., 2019). Such software tends to be less accurate for people with darker skin tones because less light reflects into the camera, making it more challenging to observe the contours of the face (cf. Buolamwini \& Gebru, 2018). Concerns also exist for complying with the Americans with Disabilities Act (ADA; 1989) — to the extent that disabilities affect speech, movement, and facial expressions, individuals protected by the ADA may be discriminated against or adversely affected by the use of AVIs. Practitioners must consider evidence beyond group means when evaluating AVI bias and discrimination, and they should carefully consider and question AVI vendors regarding how disabled applicants will be assessed.

Fourth, criterion-related validity evidence that relates AVIs to important workplace criteria such as turnover and job performance is necessary to justify their use in selection. 
Although the unitarian conception of validity values multiple sources of validity evidence, criterion-related evidence is often considered synonymous with validity. A rigorous criterion validation study first requires establishing that the focal KSAOs are job relevant. If and until such evidence is available, AVIs will be open to legal challenge. By the nature of AVIs being automated, they may receive additional scrutiny from applicants and regulators.

As mentioned above, development choices affect the psychometric properties of AVIs, although the present study is limited in its design. More research is needed, but we can offer tentative suggestions for the development of AVIs. One of the most important decisions in AVI development pertains to the ground truth. The first question to ask is, what construct(s) will be assessed? We suggest that AVIs should be developed to assess visible constructs predictive of workplace criteria. Focusing on visible constructs will enhance the availability of relevant cues. Personality traits predict performance in jobs with relevant demands (Judge \& Zapata, 2015). Yet, other constructs like cognitive ability and interpersonal skills will tend to predict job performance better than broad personality traits. Regardless of the KSAOs assessed by an AVI, organizations must use job analysis (SIOP, 2018) to determine which constructs assessed by a given AVI (if any) are relevant to the focal job.

Another important consideration beyond the choice of target construct(s) is the choice of ground truth for training the algorithms (i.e., what measure(s) of the target construct(s) should be used for model training?). The present study used observer ratings on an existing Likert-type personality scale, but using a wider variety of questions or questions that are contextualized for the context of interest may influence the accuracy and generalizability of models, which is an empirical question that remains to be tested.

One might consider training AVIs on ground truth external to the interview context- 
such as job performance. In other words, the interviews can be empirically keyed to predict job performance, and this could possibly be more resistant to faking. However, extra caution should be taken in implementing such approaches, as they may perpetuate or exacerbate demographic imbalances and past discrimination (cf. Dastin, 2018) because the model will inherit any biases in the ground truth used for training (e.g., as found in some supervisor ratings; Stauffer \& Buckley, 2005).

For some workplace relevant KSAOs, it may be beneficial to train the algorithms to model behaviorally anchored rating scale (BARS) scores from interview performance. Using BARS raises a concern regarding whether models will generalize to new interview questions. Some vendors allow question customization; others require the same set of questions to be used in all interviews; and yet others conduct local optimization of models for each focal job. In the case of models trained on BARS, AVIs may be less likely to generalize to new questions because they would be trained to model BARS anchors that describe behaviors specific to that question.

In terms of AVI-PAs, the psychometric properties for extraversion and conscientiousness models trained on interviewer-reports showed cross-sample convergence consistent with the within-sample investigations, suggesting that such models may generalize to new interview questions. These results align with the SOKA model, which states that observer-reports of more visible traits will be most predictive of relevant behaviors (Vazire, 2010). Thus, trait visibility is a necessary (but not sufficient) consideration when deciding whether client organizations can use new questions in subsequent applications of existing models trained on interviewer-reports. If a less visible trait is to be assessed, the SOKA model would suggest that self-reports will be more predictive of behaviors. Self- and interviewer-reports represent different components of personality (Hogan, 1991), so another option is to train AVI-PAs on interviewer-reports and 
have client organizations supplement the AVI-PAs with self-reports of less visible traits.

A final recommendation regards validation and reporting practices. While this study suggests that some AVI-PAs can exhibit good validity evidence for scoring some traits, "the psychometric properties of one behavior-based measure cannot be generalized to another" (Ortner \& van de Vijver, 2015, p. 7). Therefore, vendors should provide interested organizations with validation information, as required by both the Principles (SIOP, 2018) and Standards (AERA et al., 2014). This should include all of the foundational validation information, as well as the key design choices made and the rationale behind them. This should include, first, the data source (e.g., self- vs. interviewer-report) used as the "ground truth" to develop the assessment models. Second, the specific interview questions in the training data along with the rationale for the degree of interview structure (e.g., question consistency) and trait relevance should be provided. Third, if vendors allow users to tailor the interview questions, then cross-sample validation evidence must be provided.

Fourth, if vendors conduct local optimization of the algorithms, validity evidence must be generated and analyzed for the resultant, new test. Otherwise, when these procedures are eventually challenged in court, organizations may be held responsible for failing to do their due diligence when adopting AVIs.

\section{Limitations and Future Directions}

Below we highlight several directions for future research considering limitations of our current investigation. First, the scope of our research did not include AVI-PA's relationships with organizationally relevant outcomes (e.g., supervisory ratings of performance), which will be needed to justify the use of AVIs in personnel selection. Because there are additional concerns for AVIs, including their automated nature and potential for bias (Raghavan et al., 2019), it will 
be important to directly compare them to existing selection procedures. As AVIs are generally deployed as early-stage selection screening tools, they should be compared to self-report personality tests, biodata, and other forms of early-stage screening. Further, to understand if they hold potential for later stages of the selection process, they can be compared to general mental ability tests, assessment centers, and structured interviews. Criterion-related evidence of validity must be considered with other validity evidence such as provided by the present study, as well as evidence of (lack of) bias, fairness, and practical considerations in a cost-benefit analysis (SIOP, 2018) to decide whether or not adopting AVIs is justified.

Second, in some cases, the reliability of interviewer-reports in the present study was low. For instance, $\operatorname{ICC}(1, k)$ was below .60 for interviewer-reports of emotional stability in Sample 2, as well as for agreeableness, emotional stability, and openness in Sample 3. Low interrater reliability appeared to affect the psychometric properties of AVI-PAs. The within-sample investigations found that Sample 3 agreeableness models exhibited the lowest convergence of all interviewer-report models, and Samples 2 and 3 emotional stability models exhibited the next lowest convergence. Agreeableness is a highly evaluative, moderately visible trait, and emotional stability is a low visibility trait, which may have caused these low reliabilities and convergent correlations. Overall, Sample 3 had the lowest interrater reliabilities and the worst psychometric properties of all interviewer-report models in terms of construct discrimination and method variance. Therefore, collecting more raters to achieve higher reliability could lead to improved psychometric properties for interviewer-report models. However, the quality of raters and characteristics of the interview may also matter-raters in Sample 1 were I-O psychology PhD students, and although Sample 1's mock interview was relatively unstructured (i.e., gave a choice of prompts to respond to), interrater reliability was highest in Sample 1. Other rating 
formats, such as using BARS, may also bring interrater reliability more in line with metaanalytic estimates of interview interrater reliability for high structure interviews (i.e., .76; Huffcutt et al., 2013).

Third, some weaknesses in our research design limit the generalizability of our findings. Because we did not fully cross conditions relating to rater quality, question consistency, question trait relevance, and response length, the findings may be specific to our design and sample. More work is needed in the future to assess constructs with AVIs using multiple types of questions and samples to ensure that the findings generalize beyond the measures and samples used in the present study. For example, although we designed Sample 3's questions to elicit trait-relevant behaviors, we did not formally assess their trait relevance (i.e., trait activation potential).

Relatedly, we did not distinguish between generalizing across populations vs. generalizing across questions, although this may represent the real-world application of some AVIs. In the present study, Samples 2 and 3 included different interview questions while holding the population (i.e., undergraduate students) constant, whereas Sample 1 differed from Samples 2 and 3 in both interview questions and population (i.e., Turkers). This may have contributed to Sample 1's interviewer-report models exhibiting the worst convergent evidence of the interviewer-report models during the cross-sample investigations. Future research should address the extent to which the population studied, the questions used, and the interaction of the two affect the construct validity of AVIs. For instance, AVIs trained on entry-level applicants may not yield strong validity evidence when tested on C-suite applicants, even if the interview questions are held constant. Even AVIs trained on incumbents may generalize poorly to applicants due to potential range restriction in the incumbents and differences in their motivations for performing well in the interview. 
Fourth, we encourage more research to be conducted in this domain with larger samples. While the current study entails the largest number of mock video interviews to date in the domain of AVI research, it is possible that AVI-PAs could be more accurate if trained on larger samples, particularly in the case of AVI-PAs trained on self-reports (e.g., Jayaratne \& Jayatilleke, 2020). Larger samples also enable the detection of more complex relationships, such as nonlinear and interaction terms (that are included by default in the random forest algorithm; Breiman, 2001) as well as more granular trait-verbal behavior relationships in $n$-gram text mining.

Fifth, more work is needed to clarify how different modeling decisions may affect the psychometric properties of AVIs. Such investigation should include comparing results from different algorithms (e.g., random forest, support vector machines) as well as with different ways in which data are aggregated. For example, the present study used interviewee-level data (i.e., data aggregated across all interview questions) to infer interviewee characteristics, yet it would also be possible to develop question-level models using only the responses to questions meant to elicit the focal KSAO. Similarly, each question could be used to model all characteristics, and then, those predictions could be averaged together or directly evaluated. Besides different levels of analysis, methods for analyzing verbal, paraverbal, and nonverbal behavior are evolving rapidly. Linguistic analyses, especially, are growing at a rapid pace. For example, probabilistic topic models may provide interpretable methods that better capture response content (e.g., Campion et al., 2016). Recently, transfer learning and transformer language models (e.g., BERT; GPT-3) have emerged that hold promise for better capturing semantics in organizational text analysis (Hickman, Thapa, et al., 2020) and achieve high performance on a wide variety of tasks (Brown et al., 2020; Devlin et al., 2018). However, similar to how AVIs can exhibit bias, such 
transfer learning language models appear to reflect societal prejudices and biases that are embedded in the natural language texts used to develop the models (Kurita et al., 2019).

Sixth, bias concerns suggest important directions for future research: (1) investigating adverse impact and bias at both the behavioral predictor and outcome level, (2) intentionally oversampling from minority groups to ensure diversity in the training data, and (3) investigating AVIs that only use verbal behavior as predictors, considering that existing legal concerns have focused on the use of nonverbal behavior in personnel selection (e.g., EPIC, 2018) and that BARS are designed to focus on the verbal response. Initial research on written interview responses suggests that this is a promising direction (Jayaratne \& Jayatilleke, 2020).

\section{Conclusion}

Although computer scientists have provided convergent evidence of validity for AVIPAs, other important psychometric properties such as reliability, discriminant relations, content, nomological network, and generalizability were still largely unexplored. Our investigation provides initial evidence regarding the psychometric properties of AVI-PAs. Critically, the evidence for AVI-PAs trained on interviewer-reported traits generalized to new interviews, providing initial evidence that they hold promise for use in applied settings. 


\section{References}

Allport, G. W. (1937). Personality: A psychological interpretation. New York: Holt.

American Educational Research Association (AERA), American Psychological Association (APA), \& National Council on Measurement in Education (NCME). (2014). Standards for educational and psychological testing. Washington, D.C.: American Educational Research Association.

Arthur Jr, W., \& Villado, A. J. (2008). The importance of distinguishing between constructs and methods when comparing predictors in personnel selection research and practice. Journal of Applied Psychology, 93(2), 435-442.

Attali, Y. (2013). Validity and reliability of automated essay scoring. In M. D. Shermis \& J. Burstein (Eds.), Handbook of automated essay evaluation: Current applications and new directions (pp. 181-198). Taylor \& Francis.

Baltrusaitis, T., Zadeh, A., Lim, Y. C., \& Morency, L. P. (2018, May). Openface 2.0: Facial behavior analysis toolkit. In 2018 13th IEEE International Conference on Automatic Face \& Gesture Recognition (FG 2018) (pp. 59-66). IEEE.

Barrett, L. F., Adolphs, R., Marsella, S., Martinez, A. M., \& Pollak, S. D. (2019). Emotional Expressions Reconsidered: Challenges to Inferring Emotion From Human Facial Movements. Psychological Science in the Public Interest, 20(1), 1-68. https://doi.org/10.1177/1529100619832930

Barrick, M. R., \& Mount, M. K. (1991). The big five personality dimensions and job performance: A meta-analysis. Personnel Psychology, 44(1), 1-26. https://doi.org/10.1111/j.1744-6570.1991.tb00688.x

Barrick, M. R., Swider, B. W., \& Stewart, G. L. (2010). Initial Evaluations in the Interview: Relationships with Subsequent Interviewer Evaluations and Employment Offers. Journal of Applied Psychology, 95(6), 1163-1172. https://doi.org/10.1037/a0019918

Bell, T. (2019, January 15). This bot judges how much you smile during your job interview. https://www.fastcompany.com/90284772/this-bot-judges-how-much-you-smile-duringyour-job-interview

Biel, J.-I., Tsiminaki, V., Dines, J., \& Gatica-Perez, D. (2013). Hi YouTube! Personality impressions and verbal content in social video. In International Conference on Multimodal Interaction (ICMI'13) (pp. 119-126). https://doi.org/10.1145/2522848.2522877

Blackman, M. C. (2002). Personality judgment and the utility of the unstructured employment interview. Basic and Applied Social Psychology, 24(3), 241-250. https://doi.org/10.1207/S15324834BASP2403_6

Bleidorn, W., \& Hopwood, C. J. (2019). Using Machine Learning to Advance Personality Assessment and Theory. Personality and Social Psychology Review, 23(2), 190-203. 
https://doi.org/10.1177/1088868318772990

Bommer, W. H., Johnson, J. L., Rich, G. A., Podsakoff, P. M., \& MacKenzie, S. B. (1995). On the interchangeability of objective and subjective measures of employee performance: A meta-analysis. Personnel Psychology, 48(3), 587-605.

Bosch, N., \& D'Mello, S. (2019). Automatic detection of mind wandering from video in the lab and in the classroom. IEEE Transactions on Affective Computing. https://doi.org/10.1109/TAFFC.2019.2908837

Bosco, F. A., Aguinis, H., Singh, K., Field, J. G., \& Pierce, C. A. (2015). Correlational effect size benchmarks. Journal of Applied Psychology, 100(2), 431-449. https://doi.org/10.1037/a0038047

Bourdage, J. S., Roulin, N., \& Tarraf, R. (2018). "I (might be) just that good": Honest and deceptive impression management in employment interviews. Personnel Psychology, (July 2016), 1-36. https://doi.org/10.1111/peps.12285

Breiman, L. (2001). Random forests. Machine Learning, 45(1), 5-32. https://doi.org/10.1023/A:1010933404324

Brown, T. B., Mann, B., Ryder, N., Subbiah, M., Kaplan, J., Dhariwal, P., ... \& Agarwal, S. (2020). Language models are few-shot learners. arXiv preprint arXiv:2005.14165.

Buolamwini, J., \& Gebru, T. (2018, January). Gender shades: Intersectional accuracy disparities in commercial gender classification. In Conference on fairness, accountability and transparency (pp. 77-91).

Campbell, D. T., \& Fiske, D. (1959). Convergent and discriminant validation by the multitraitmultimethod matrix. Psychological Bulletin, 59(2), 81-105. Retrieved from http://www.ncbi.nlm.nih.gov/pubmed/19586159

Campion, M. A., Palmer, D. K., \& Campion, J. E. (1997). A review of structure in the selection interview. Personnel Psychology, 50(3), 655-702. https://doi.org/10.1111/j.17446570.1997.tb00709.x

Campion, M. C., Campion, M. A., Campion, E. D., \& Reider, M. H. (2016). Initial investigation into computer scoring of candidate essays for personnel selection. Journal of Applied Psychology, 101(7), 958-975. https://doi.org/10.1037/ap10000108

Chamorro-Premuzic, T., Akhtar, R., Winsborough, D., \& Sherman, R. A. (2017). The datafication of talent: how technology is advancing the science of human potential at work. Current Opinion in Behavioral Sciences, 18, 13-16. https://doi.org/10.1016/j.cobeha.2017.04.007

Chamorro-Premuzic, T., Winsborough, D., Sherman, R. A., \& Hogan, R. (2016). New talent signals: Shiny new objects or a brave new world? Industrial and Organizational Psychology, 9(3), 621-640. https://doi.org/10.1017/iop.2016.6 
Chapman, B. P., Weiss, A., \& Duberstein, P. R. (2016). Statistical learning theory for high dimensional prediction: Application to criterion-keyed scale development. Psychological Methods, 21(4), 603-620. https://doi.org/10.1037/met0000088

Chapman, D. S., \& Zweig, D. I. (2005). Developing a nomological network for interview structure: Antecedents and consequences of the structured selection interview. Personnel Psychology, 58(3), 673-702. https://doi.org/10.1111/j.1744-6570.2005.00516.x

Chen, L., Feng, G., Leong, C. W., Lehman, B., Martin-Raugh, M., Kell, H., ... Yoon, S.-Y. (2016). Automated scoring of interview videos using Doc2Vec multimodal feature extraction paradigm. Proceedings of the 18th ACM International Conference on Multimodal Interaction - ICMI 2016, 161-168. https://doi.org/10.1145/2993148.2993203

Chen, L., Zhao, R., Leong, C. W., Lehman, B., Feng, G., \& Hoque, M. (2018). Automated video interview judgment on a large-sized corpus collected online. 2017 7th International Conference on Affective Computing and Intelligent Interaction, ACII 2017, 504-509. https://doi.org/10.1109/ACII.2017.8273646

Christiansen, N. D., Wolcott-Burnam, S., Janovics, J. E., Burns, G. N., \& Quirk, S. W. (2005). The good judge revisited: Individual differences in the accuracy of personality judgments. Human Performance, 18(2), 123-149.

Connelly, B. S., \& Ones, D. S. (2010). An other perspective on personality: Meta-analytic integration of observers' accuracy and predictive validity. Psychological Bulletin, 136(6), 1092-1122. https://doi.org/10.1037/a0021212

Cortina, J. M., Goldstein, N. B., Payne, S. C., Davison, H. K., \& Gilliland, S. W. (2000). The incremental validity of interview scores over and above cognitive ability and conscientiousness scores. Personnel Psychology, 53, 325-351.

Cronbach, L. J. (1990). Essentials of Psychological Testing (Fifth). New York: Harper \& Row.

Cucina, J. M., Vasilopoulos, N. L., Su, C., Busciglio, H. H., Cozma, I., DeCostanza, A. H., ... Shaw, M. N. (2019). The Effects of Empirical Keying of Personality Measures on Faking and Criterion-Related Validity. Journal of Business and Psychology, 34(3), 337-356. https://doi.org/10.1007/s10869-018-9544-y

Cuddy, A. J. C., Wilmuth, C. A., Yap, A. J., \& Carney, D. R. (2015). Preparatory power posing affects nonverbal presence and job interview performance. Journal of Applied Psychology, 100(4), 1286-1295. https://doi.org/10.1037/a0038543

Dastin, J. (2018). Amazon scraps secret AI recruiting tool that showed bias against women. Reuters, October 2018.

De Kock, F. S., Lievens, F., \& Born, M. P. (2015). An in-depth look at dispositional reasoning and interviewer accuracy. Human Performance, 28(3), 199-221.

DeGroot, T., \& Gooty, J. (2009). Can nonverbal cues be used to make meaningful personality 
attributions in employment interviews? Journal of Business and Psychology, 24(2), 179192. https://doi.org/10.1007/s10869-009-9098-0

DeGroot, T., \& Kluemper, D. (2007). Evidence of predictive and incremental validity of personality factors, vocal attractiveness and the situational interview. International Journal of Selection and Assessment, 15(1), 30-39. https://doi.org/10.1111/j.14682389.2007.00365.x

Devlin, J., Chang, M. W., Lee, K., \& Toutanova, K. (2018). Bert: Pre-training of deep bidirectional transformers for language understanding. arXiv preprint arXiv:1810.04805.

Ekman, P., \& Friesen, W. V. (1978). Facial Action Coding System: A technique for the measurement of facial action. Palo Alto, CA: Consulting Psychologists Press.

Electronic Privacy Information Center (EPIC). (2019). Complaint and request for investigation, injunction, and other relief. https://www.washingtonpost.com/context/epic-s-ftc-complaintabout-hirevue/9797b738-e36a-4b7a-8936-667cf8748907/

Eyben, F. (2014). Real-time speech and music classification by large audio feature space extraction. https://doi.org/10.1007/978-3-319-27299-3

Eyben, F., Scherer, K. R., Schuller, B. W., Sundberg, J., Andre, E., Busso, C., ... Truong, K. P. (2016). The Geneva Minimalistic Acoustic Parameter Set (GeMAPS) for Voice Research and Affective Computing. IEEE Transactions on Affective Computing, 7(2), 190-202. https://doi.org/10.1109/TAFFC.2015.2457417

Feiler, A. R., \& Powell, D. M. (2016). Behavioral Expression of Job Interview Anxiety. Journal of Business and Psychology, 31(1), 155-171. https://doi.org/10.1007/s10869-015-9403-z

Feinerer, I., Hornik, K., \& Meyer, D. (2008). Text mining infrastructure in R. Journal of Statistical Software, 25(5), 1-54.

Funder, D. C. (1995). On the accuracy of personality judgment: A realistic approach. Psychological Review, 102(4), 652-670.

Funder, D. C. (2012). Accurate Personality Judgment. Current Directions in Psychological Science, 21(3), 177-182. https://doi.org/10.1177/0963721412445309

Funder, D. C., \& West, S. G. (1993). Consensus, self-other agreement, and accuracy in personality judgment: An introduction. Journal of Personality, 61(4), 457-476. https://doi.org/10.1111/j.1467-6494.1993.tb00778.x

Goldberg, L. R. (1992). The development of markers for the big-five factor structure. Psychological Assessment, 4(1), 26-42.

Goldberg, L. R. (1999). A broad-bandwidth, public domain, personality inventory measuring the lower-level facets of several five-factor models. Personality Psychology in Europe, 7(1), 728. 
Goodfellow, I. J., Shlens, J., \& Szegedy, C. (2014). Explaining and harnessing adversarial examples. ArXiv Preprint ArXiv:1412.6572.

Gosling, S. D., Rentfrow, P. J., \& Swann Jr., W. B. (2003). A very brief measure of the Big-Five personality domains. Journal of Research in Personality, 37(6), 504-528. https://doi.org/10.1016/S0092-6566(03)00046-1

Hamdani, M. R., Valcea, S., \& Buckley, M. R. (2014). The relentless pursuit of construct validity in the design of employment interviews. Human Resource Management Review, 24(2), 160-176. https://doi.org/10.1016/j.hrmr.2013.07.002

Harris, K. D., Murray, P., \& Warren, E. (2019). Letter to U.S. Equal Employment Opportunity Commission regarding risks of facial recognition technology. Retrieved from https://www.scribd.com/document/388920670/SenHarris-EEOC-Facial-Recognition-2

Harwell, D. (2019, November 6). A face-scanning algorithm increasingly decides whether you deserve the job. https://www.washingtonpost.com/technology/2019/10/22/ai-hiring-facescanning-algorithm-increasingly-decides-whether-you-deserve-job/

Hickman, L., Saef, R., Ng, V., Tay, L., Woo, S. E., \& Bosch, N. (2020). Developing and evaluating language-based machine learning algorithms for inferring applicant personality in video interviews. Manuscript submitted for publication.

Hickman, L., Tay, L., \& Woo, S. E. (2019). Validity investigation of off-the-shelf languagebased personality assessment using video interviews: Convergent and discriminant relationships with self and observer ratings. Personnel Assessment and Decisions, 5(3), 1220. https://doi.org/10.25035/pad.2019.03.003

Hickman, L., Thapa, S., Tay, L., Cao, M., \& Srinivasan, P. (2020). Text preprocessing for text mining in organizational research: Review and recommendations. Organizational Research Methods, 1-33. https://doi.org/10.1177/1094428120971683

Hogan, R. T. (1991). Personality and personality measurement. In M. D. Dunnette \& L. M. Hough (Eds.), Handbook of Industrial and Organizational Psychology (Vol. 2) (2nd ed., pp. 873-919). Palo Alto, CA: Consulting Psychologists Press, Inc.

Huffcutt, A. I., Conway, J. M., Roth, P. L., \& Stone, N. J. (2001). Identification and MetaAnalytic Assessment of Psychological Constructs Measured in Employment Interviews. Journal of Applied Psychology, 86(5), 897-913.

Huffcutt, A. I., Culbertson, S. S., \& Weyhrauch, W. S. (2013). Employment interview reliability: New meta-analytic estimates by structure and format. International Journal of Selection and Assessment, 21(3), 264-276. https://doi.org/10.1111/ijsa.12036

Huffcutt, A. I., Van Iddekinge, C. H., \& Roth, P. L. (2011). Understanding applicant behavior in employment interviews: A theoretical model of interviewee performance. Human Resource Management Review, 21(4), 353-367. 
IBM. (2019). IBM Watson Speech to Text. Available at https://www.ibm.com/watson/services/speech-to-text/

Ickes, W. (2016). Empathic accuracy: Judging thoughts and feelings. In J. A. Hall, M. Schmid Mast, \& T. V. West (Eds.), The social psychology of perceiving others accurately (pp. 5270). Cambridge University Press.

Jayaratne, M., \& Jayatilleke, B. (2020). Predicting personality using answers to open-ended interview questions. IEEE Access, 8, 115345-115355.

John, O. P., \& Robins, R. W. (1993). Determinants of interjudge agreement on personality traits: The Big Five domains, observability, evaluativeness, and the unique perspective of the self. Journal of Personality, 61(4), 521-551.

Judge, T. A., \& Zapata, C. P. (2015). The person-situation debate revisited: Effect of situation strength and trait activation on the validity of the big five personality traits in predicting job performance. Academy of Management Journal, 58(4), 1149-1179. https://doi.org/10.5465/amj.2010.0837

Kenny, D. A. (1991). A general model of consensus and accuracy in interpersonal perception. Psychological Review, 98(2), 155-163. https://doi.org/10.1037//0033-295x.98.2.155

Kenny, D. A. (2004). PERSON: A general model of interpersonal perception. Personality and Social Psychology Review, 8(3), 265-280. https://doi.org/10.1207/s15327957pspr0803_3

Kim, H., Di Domenico, S. I., \& Connelly, B. S. (2019). Self-other agreement in personality reports: A meta-analytic comparison of self-and informant-report means. Psychological Science, 30(1), 129-138.

Kristof-Brown, A., Barrick, M. R., \& Franke, M. (2002). Applicant impression management: Dispositional influences and consequences for recruiter perceptions of fit and similarity. Journal of Management, 28(1), 27-46. https://doi.org/10.1016/S0149-2063(01)00131-3

Kuhn, M. (2008). Building predictive models in R using the caret package. Journal of Statistical Software, 28(5), 1-26. https://doi.org/10.1053/j.sodo.2009.03.002

Kuncel, N. R., Credé, M., \& Thomas, L. L. (2005). The validity of self-reported grade point averages, class ranks, and test scores: A meta-analysis and review of the literature. Review of Educational Research, 75(1), 63-82. https://doi.org/10.3102/00346543075001063

Kurita, K., Vyas, N., Pareek, A., Black, A. W., \& Tsvetkov, Y. (2019). Measuring bias in contextualized word representations. arXiv preprint arXiv:1906.07337.

Langer, M., König, C. J., \& Papathanasiou, M. (2019). Highly automated job interviews: Acceptance under the influence of stakes. International Journal of Selection and Assessment, 27(3), 217-234.

Le, H., Schmidt, F. L., \& Putka, D. J. (2009). The multifaceted nature of measurement artifacts 
and its implications for estimating construct-level relationships. Organizational Research Methods, 12(1), 165-200.

Lievens, F., Chasteen, C. S., Day, E. A., \& Christiansen, N. D. (2006). Large-scale investigation of the role of trait activation theory for understanding assessment center convergent and discriminant validity. Journal of Applied Psychology, 91(2), 247-258. https://doi.org/10.1037/0021-9010.91.2.247

Lin, J. (1991). Divergence measures based on the Shannon entropy. IEEE Transactions on Information Theory, 37(1), 145-151.

Lord, F. M., \& Novick, M. R. (1968). Statistical theories of mental test scores. Reading, MA: Addison-Wesley.

Madera, J. M., \& Hebl, M. R. (2012). Discrimination against facially stigmatized applicants in interviews: An eye-tracking and face-to-face investigation. Journal of Applied Psychology, 97(2), 317-330. https://doi.org/10.1037/a0025799

McCrae, R. R., Kurtz, J. E., Yamagata, S., \& Terracciano, A. (2011). Internal Consistency, Retest Reliability, and their implications. Personality and Social Psychological Bulletin, 15(1), 28-50. https://doi.org/10.1177/1088868310366253.Internal

McGraw, K. O., \& Wong, S. P. (1996). Forming inferences about some intraclass correlation coefficients. Psychological Methods, 1(1), 30-46. https://doi.org/10.1037/1082-989X.1.1.30

Messick, S. (1989). Meaning and Values in Test Validation: The Science and Ethics of Assessment. Educational Researcher, 18(2), 5-11. https://doi.org/10.3102/0013189X018002005

Messinger, D. S., Fogel, A., \& Dickson, K. (2001). All smiles are positive, but some smiles are more positive than others. Developmental Psychology, 37(5), 642-653.

Mulfinger, E., Wu, F., Alexander, L., III, \& Oswald, F. L. (2020, February). AI technologies in talent management systems: It glitters but is it gold? Poster presented at Work in the $21^{\text {st }}$ Century: Automation, Workers, and Society, Houston, TX.

Mischel, W., \& Shoda, Y. (1995). A cognitive-affective system theory of personality: reconceptualizing situations, dispositions, dynamics, and invariance in personality structure. Psychological Review, 102(2), 246-268.

Morgeson, F. P., Campion, M. A., Dipboye, R. L., Hollenbeck, J. R., Murphy, K., \& Schmitt, N. (2007). Reconsidering the use of personality tests in personnel selection contexts. Personnel ..., 60, 683-729. Retrieved from http://onlinelibrary.wiley.com/doi/10.1111/j.17446570.2007.00089.x/full

Naim, I., Tanveer, I., Gildea, D., \& Hoque, M. E. (2018). Automated Analysis and Prediction of Job Interview Performance. IEEE Transactions on Affective Computing, 9(2), 191-204. https://doi.org/10.1109/TAFFC.2016.2614299 
Nguyen, L. S., Frauendorfer, D., Mast, M. S., \& Gatica-Perez, D. (2014). Hire me:

Computational inference of hirability in employment interviews based on nonverbal behavior. IEEE Transactions on Multimedia, 16(4), 1018-1031. https://doi.org/10.1109/TMM.2014.2307169

Nguyen, L. S., \& Gatica-Perez, D. (2016). Hirability in the Wild: Analysis of Online Conversational Video Resumes. IEEE Transactions on Multimedia, 18(7), 1422-1437. https://doi.org/10.1109/TMM.2016.2557058

Noftle, E. E., \& Robins, R. W. (2007). Personality predictors of academic outcomes: Big five correlates of GPA and SAT scores. Journal of Personality and Social Psychology, 93(1), 116-130. https://doi.org/10.1037/0022-3514.93.1.116

Ortner, T. M., \& van de Vijver, F. J. R. (2015). Assessment beyond self-reports. In T. M. Ortner \& F. J. R. van de Vijver (Eds.), Behavior-based assessment in psychology: Going beyond self-report in the personality, affective, motivation, and social domains (pp. 3-14). Hogrefe Publishing.

Oswald, F. L., Behrend, T. S., Putka, D. J., \& Sinar, E. (2020). Big Data in IndustrialOrganizational Psychology and Human Resource Management: Forward Progress for Organizational Research and Practice. Annual Review of Organizational Psychology and Organizational Behavior, 7(1). https://doi.org/10.1146/annurev-orgpsych-032117-104553

Park, G., Schwartz, H. A., Eichstaedt, J. C., Kern, M. L., Kosinski, M., Stillwell, D. J., ... Seligman, M. E. P. (2015). Automatic personality assessment through social media language. Journal of Personality and Social Psychology, 108(6), 934-952. https://doi.org/10.1037/pspp0000020

Pedregosa, F., Varoquaux, G., Gramfort, A., Michel, V., Thirion, B., Grisel, O., ... \& Vanderplas, J. (2011). Scikit-learn: Machine learning in Python. The Journal of Machine Learning Research, 12, 2825-2830.

Peeters, H., \& Lievens, F. (2006). Verbal and nonverbal impression management tactics in behavior description and situational interviews. International Journal of Selection and Assessment, 14(3), 206-222. https://doi.org/10.1111/j.1468-2389.2006.00348.x

Pennebaker, J. W., Boyd, R. L., Jordan, K., \& Blackburn, K. (2015). The development and psychometric properties of LIWC2015. University of Texas at Austin.

Ployhart, R. E., Schmitt, N., \& Tippins, N. T. (2017). Solving the Supreme Problem: 100 Years of selection and recruitment at the Journal of Applied Psychology. Journal of Applied Psychology, 102(3), 291-304. https://doi.org/10.1037/ap10000081

Ponce-López, V., Chen, B., Oliu, M., Corneanu, C., Clapés, A., Guyon, I., ... Escalera, S. (2016). Chalearn LAP 2016: First round challenge on first impressions - Dataset and results. Lecture Notes in Computer Science, 9915 LNCS(October), 400-418. https://doi.org/10.1007/978-3-319-49409-8_32 
Poropat, A. E. (2009). A meta-analysis of the five-factor model of personality and academic performance. Psychological Bulletin, 135(2), 322-338. https://doi.org/10.1037/a0014996

Powell, D. M., \& Bourdage, J. S. (2016). The detection of personality traits in employment interviews: Can "good judges" be trained?. Personality and Individual Differences, 94, 194199.

Putka, D. J., Beatty, A. S., \& Reeder, M. C. (2018). Modern Prediction Methods: New Perspectives on a Common Problem. Organizational Research Methods, 21(3), 689-732. https://doi.org/10.1177/1094428117697041

Raghavan, M., Barocas, S., Kleinberg, J., \& Levy, K. (2020). Mitigating bias in algorithmic employment screening: Evaluating claims and practices. In 2020 Conference on Fairness, Accountability, and Transparency (FAT) (pp. 469-481). https://doi.org/10.2139/ssrn.3408010

Raykov, T., Marcoulides, G. A., \& Tong, B. (2016). Do two or more multicomponent instruments measure the same construct? Testing construct congruence using latent variable modeling. Educational and Psychological Measurement, 76(5), 873-884. https://doi.org/10.1177/0013164415604705

Rotolo, C. T., Church, A. H., Adler, S., Smither, J. W., Colquitt, A. L., Shull, A. C., ... Foster, G. (2018). Putting an End to Bad Talent Management: A Call to Action for the Field of Industrial and Organizational Psychology. Industrial and Organizational Psychology, 11(2), 176-219. https://doi.org/10.1017/iop.2018.6

Sajjadiani, S., Sojourner, A. J., Kammeyer-Mueller, J. D., \& Mykerezi, E. (2019). Using Machine Learning to Translate Applicant Work History Into Predictors of Performance and Turnover. Journal of Applied Psychology. https://doi.org/10.1037/ap10000405

Schleicher, D. J., Van Iddekinge, C. H., Morgeson, F. P., \& Campion, M. A. (2010). If at first you don't succeed, try, try again: Understanding race, age, and gender differences in retesting score improvement. Journal of Applied Psychology, 95(4), 603-617. https://doi.org/10.1037/a0018920

Shaffer, J. A., DeGeest, D., \& Li, A. (2016). Tackling the problem of construct proliferation: A guide to assessing the discriminant validity of conceptually related constructs. Organizational Research Methods, 19(1), 80-110. https://doi.org/10.1177/1094428115598239

Shaffer, J. A., \& Postlethwaite, B. E. (2012). A matter of context: A meta-analytic investigation of the relative validity of contextualized and noncontextualized personality measures. Personnel Psychology, 65(3), 445-493. https://doi.org/10.1111/j.1744-6570.2012.01250.x

Simms, L. J. (2008). Classical and modern methods of psychological scale construction. Social and Personality Psychology Compass, 2(1), 414-433. https://doi.org/10.1111/j.17519004.2007.00044.x 
Society for Industrial and Organizational Psychology (SIOP). (2018). Principles for the Validation and Use of Personnel Selection Procedures (Fifth). American Psychological Association. https://doi.org/10.1017/iop.2018.195

Speer, A. B. (2018). Quantifying with words: An investigation of the validity of narrativederived performance scores. Personnel Psychology, 71(3), 299-333. https://doi.org/10.1111/peps.12263

Stauffer, J. M., \& Buckley, M. R. (2005). The Existence and Nature of Racial Bias in Supervisory Ratings. Journal of Applied Psychology, 90(3), 586-591. https://doi.org/10.1037/0021-9010.90.3.586

Swider, B. W., Barrick, M. R., \& Brad Harris, T. (2016). Initial impressions: What they are, what they are not, and how they influence structured interview outcomes. Journal of Applied Psychology, 101(5), 625-638. https://doi.org/10.1037/ap10000077

Swider, B. W., Barrick, M. R., Harris, T. B., \& Stoverink, A. C. (2011). Managing and creating an image in the interview: The role of interviewee initial impressions. Journal of Applied Psychology, 96(6), 1275-1288. https://doi.org/10.1037/a0024005

Tay, L., Woo, S. E., Hickman, L., \& Saef, R. (2020). Psychometric and validity issues in machine learning approaches to personality assessment: A focus on social media text mining. European Journal of Personality, 1-19. https://doi.org/10.1002/per.2290

Tellegen, A. (1991). Personality traits: Issues of definition, evidence, and assessment. In Thinking clearly about psychology: Essays in honor of Paul E. Meehl, Vol. 1: Matters of public interest (pp. 10-35). Minneapolis: University of Minnesota Press.

Tett, R. P., \& Burnett, D. D. (2003). A personality trait-based interactionist model of job performance. The Journal of Applied Psychology, 88(3), 500-517. https://doi.org/10.1037/0021-9010.88.3.500

Tett, R. P., \& Guterman, H. A. (2000). Situation trait relevance, trait expression, and crosssituational consistency: Testing a principle of trait activation. Journal of Research in Personality, 34(4), 397-423. https://doi.org/10.1006/jrpe.2000.2292

Van Iddekinge, C. H., Raymark, P. H., \& Roth, P. L. (2005). Assessing personality with a structured employment interview: Construct-related validity and susceptibility to response inflation. Journal of Applied Psychology, 90(3), 536-552. https://doi.org/10.1037/00219010.90.3.536

Vazire, S. (2010). Who knows what about a person? The self-other knowledge asymmetry (SOKA) model. Journal of Personality and Social Psychology, 98(2), 281-300. https://doi.org/10.1037/a0017908

Woehr, D. J., Putka, D. J., \& Bowler, M. C. (2012). An examination of g-theory methods for modeling multitrait-multimethod data: Clarifying links to construct validity and confirmatory factor analysis. Organizational Research Methods, 15(1), 134-161. 
https://doi.org/10.1177/1094428111408616

Woo, S. E., Jin, J., \& LeBreton, J. M. (2015). Specificity Matters: Criterion-Related Validity of Contextualized and Facet Measures of Conscientiousness in Predicting College Student Performance. Journal of Personality Assessment, $0(0), 1-9$. https://doi.org/10.1080/00223891.2014.1002134

Wood, D., Harms, P., \& Vazire, S. (2010). Perceiver effects as projective tests: What your perceptions of others say about you. Journal of Personality and Social Psychology, 99(1), 174-190. https://doi.org/10.1037/a0019390

Zou, H., \& Hastie, T. (2005). Regularization and variable selection via the elastic net. Journal of the Royal Statistical Society. Series B: Statistical Methodology, 67(2), 301-320. https://doi.org/10.1111/j.1467-9868.2005.00527.x 
Appendices

Table A1

Within-sample 10-fold cross-validated convergence Sample 1 Sample 2 Sample

3

Self-reports

$\begin{array}{lllllll}r & \rho & r & P & r & \rho & \bar{r}\end{array}$

$\begin{array}{llllllll}\text { Extraversion } & .09 & .09 & .30 & .32 & .01 & .01 & .13\end{array}$

$\begin{array}{lllllllll}\text { Agreeableness } & -.08 & -.09 & .25 & .27 & .16 & .18 & .11\end{array}$

$\begin{array}{llllllll}\text { Conscientiousness } & -.05 & -.05 & .05 & .06 & .02 & .02 & .01\end{array}$

$\begin{array}{llllllll}\text { Emotional Stability } & .28 & .29 & .05 & .05 & .31 & .33 & .21\end{array}$

$\begin{array}{llllllll}\text { Openness } & .05 & .05 & -.11 & -.13 & .25 & .29 & .06\end{array}$

Interviewer-reports

$\begin{array}{llllllll}\text { Extraversion } & .65 & .75 & .65 & .69 & .65 & .74 & .65 \\ \text { Agreeableness } & .41 & .48 & .44 & .56 & .17 & .22 & .34 \\ \text { Conscientiousness } & .41 & .46 & .41 & .53 & .43 & .53 & .42 \\ \text { Emotional Stability } & .32 & .40 & .24 & .32 & .25 & .35 & .27 \\ \text { Openness } & .27 & .33 & .37 & .44 & .41 & .59 & .35\end{array}$

Note. $r=$ mean observed correlation between predicted and actual traits averaged across the 10 folds. $\rho=$ mean observed correlation corrected for unreliability (using Cronbach's alpha for self-reports and interrater reliability for interviewer-reports). 
Table A2

Cross-sample convergence

Self-reports

Extraversion

Agreeableness

.07

.10

.04

.07

Conscientiousness

.08

.10

$.09 \quad .09$

Emotional Stability

.01

.06

$.06 \quad .04$

Openness

.04

.14

$.13 \quad .10$

Interviewer-reports

Extraversion

.64

.04

.04

.07

Agreeableness

.28

.65

.63

.64

Conscientiousness

.34

.28

.22

.26

Emotional Stability

.18

.52

.45

.44

Openness

.24

.24

.22

.21

Note: $r=$ the sample size weighted mean observed correlation between cross-sample predictions and reported traits. $\bar{r}=$ mean observed correlation across the six cross-sample predictions and reported traits. 
Table A3

Multitrait-multimethod matrix of Sample 1 AVI-PA scores and interviewer-reported traits in Samples $2 \& 3$

\begin{tabular}{lrrrrrrrrrr}
\hline & 1 & 2 & 3 & 4 & 5 & 6 & 7 & 8 & 9 & 10 \\
\hline AVI-based & & & & & & & & & & \\
1. Extraversion & - & .26 & .21 & .28 & .17 & $\mathbf{. 6 4}$ & .19 & .28 & .19 & .30 \\
2. Agreeableness & .26 & - & .10 & .14 & .09 & .25 &. $\mathbf{3 2}$ & .17 & .12 & .07 \\
3. Conscientiousness & .34 & .14 & - & .25 & .11 & .17 & .04 &. $\mathbf{3 2}$ & .14 & .13 \\
4. Emotional stability & .27 & .09 & .40 & - & .02 & .21 & .04 & .21 &. $\mathbf{1 3}$ & .11 \\
5. Openness & .21 & .13 & .25 & .10 & - & .14 & .16 & .09 & .06 & .22 \\
Interviewer-reports & & & & & & & & & & \\
6. Extraversion &. $\mathbf{6 3}$ & .25 & .38 & .24 & .27 & - & .29 & .29 & .40 & .43 \\
7. Agreeableness & .21 &. $\mathbf{2 4}$ & .14 & .12 & .12 & .30 & - & .14 & .24 & .17 \\
8. Conscientiousness & .28 & .11 &. $\mathbf{3 7}$ & .31 & .11 & .27 & .34 & - & .35 & .38 \\
9. Emotional stability & -.12 & .07 & -.21 &. $\mathbf{2 4}$ & .07 & .20 & .42 & .45 & - & .36 \\
10. Openness & .30 & .19 & .17 & .13 &. $\mathbf{2 6}$ & .45 & .34 & .41 & .20 & - \\
\hline
\end{tabular}

Note: AVI-based = automated video interview personality assessments. Sample 2 correlations are above the diagonal, and Sample 3 correlations are below the diagonal. Convergent correlations are in bold.

Table A4

Multitrait-multimethod matrix of Sample 2 AVI-PA scores and interviewer-reported personality in Samples 1 \& 3

\begin{tabular}{llllllllll}
1 & 2 & 3 & 4 & 5 & 6 & 7 & 8 & 9 & 10 \\
\hline
\end{tabular}

AVI-based

\begin{tabular}{lrrrrrrrrrr} 
1. Extraversion & - & .24 & .51 & .42 & .55 & $\mathbf{. 6 4}$ & .12 & .18 & .19 & .14 \\
2. Agreeableness & .11 & - & .06 & .14 & .07 & .26 &. $\mathbf{2 8}$ & -.01 & .00 & .01 \\
3. Conscientiousness & .61 & .10 & - & .40 & .49 & .35 & .17 & $\mathbf{. 5 0}$ & .33 & .22 \\
4. Emotional stability & .37 & .16 & .53 & - & .39 & .26 & .08 & .21 & $\mathbf{. 2 1}$ & .15 \\
5. Openness & .61 & -.06 & .52 & .23 & - & .35 & .12 & .25 & .15 &. $\mathbf{3 5}$ \\
Interviewer-reports & & & & & & & & & & \\
6. Extraversion & .66 & .19 & .54 & .37 & .45 & - & .21 & .21 & .28 & .26 \\
7. Agreeableness & .15 & .28 & .30 & .16 & .13 & .30 & - & .34 & .33 & .25 \\
8. Conscientiousness & .26 & .03 & .54 & .32 & .36 & .27 & .34 & - & .44 & .19 \\
9. Emotional stability & .10 & .00 & .29 &. $\mathbf{2 8}$ & .12 & .20 & .42 & .45 & - & .11 \\
10. Openness & .30 & .14 & .36 & .16 & $\mathbf{. 3 4}$ & .45 & .34 & .41 & .20 & - \\
\hline
\end{tabular}

Note: AVI-based = automated video interview personality assessments. Sample 1 correlations are above the diagonal, and Sample 3 correlations are below the diagonal. Convergent correlations are in bold. 
Table A5

Multitrait-multimethod matrix of Sample 3 AVI-PA scores and interviewer-reported personality in Samples $1 \& 2$

\begin{tabular}{lrrrrrrrrrr}
\hline & 1 & 2 & 3 & 4 & 5 & 6 & 7 & 8 & 9 & 10 \\
\hline AVI-based & & & & & & & & & & \\
1. Extraversion & - & .48 & .56 & .14 & .62 &. $\mathbf{5 9}$ & .13 & .28 & .22 & .16 \\
2. Agreeableness & .40 & - & .52 & .17 & .50 & .34 &. $\mathbf{2 6}$ & .28 & .26 & .07 \\
3. Conscientiousness & .58 & .40 & - & .31 & .61 & .29 & .16 &. $\mathbf{4 3}$ & .31 & .19 \\
4. Emotional stability & .15 & .12 & .34 & - & .10 & .12 & .06 & .19 &. $\mathbf{2 6}$ & .22 \\
5. Openness & .68 & .44 & .56 & .08 & - & .42 & .22 & .31 & .27 &. $\mathbf{2 3}$ \\
Interviewer-reports & & & & & & & & & & \\
6. Extraversion & .65 & .25 & .37 & .10 & .46 & - & .21 & .21 & .28 & .26 \\
7. Agreeableness & .17 &. $\mathbf{1 8}$ & .10 & -.12 & .15 & .29 & - & .34 & .33 & .25 \\
8. Conscientiousness & .26 & .19 &. $\mathbf{4 6}$ & .18 & .32 & .29 & .14 & - & .44 & .19 \\
9. Emotional stability & .22 & .12 & .27 &. $\mathbf{2 0}$ & .17 & .40 & .24 & .35 & - & .11 \\
10. Openness & .29 & .07 & .25 & .07 &. $\mathbf{3 0}$ & .43 & .17 & .38 & .36 & - \\
\hline
\end{tabular}

Note: AVI-based = automated video interview personality assessments. Sample 1 correlations are above the diagonal, and Sample 2 correlations are below the diagonal. Convergent correlations are in bold. 


\section{Online Supplement: Nested cross-validation code}

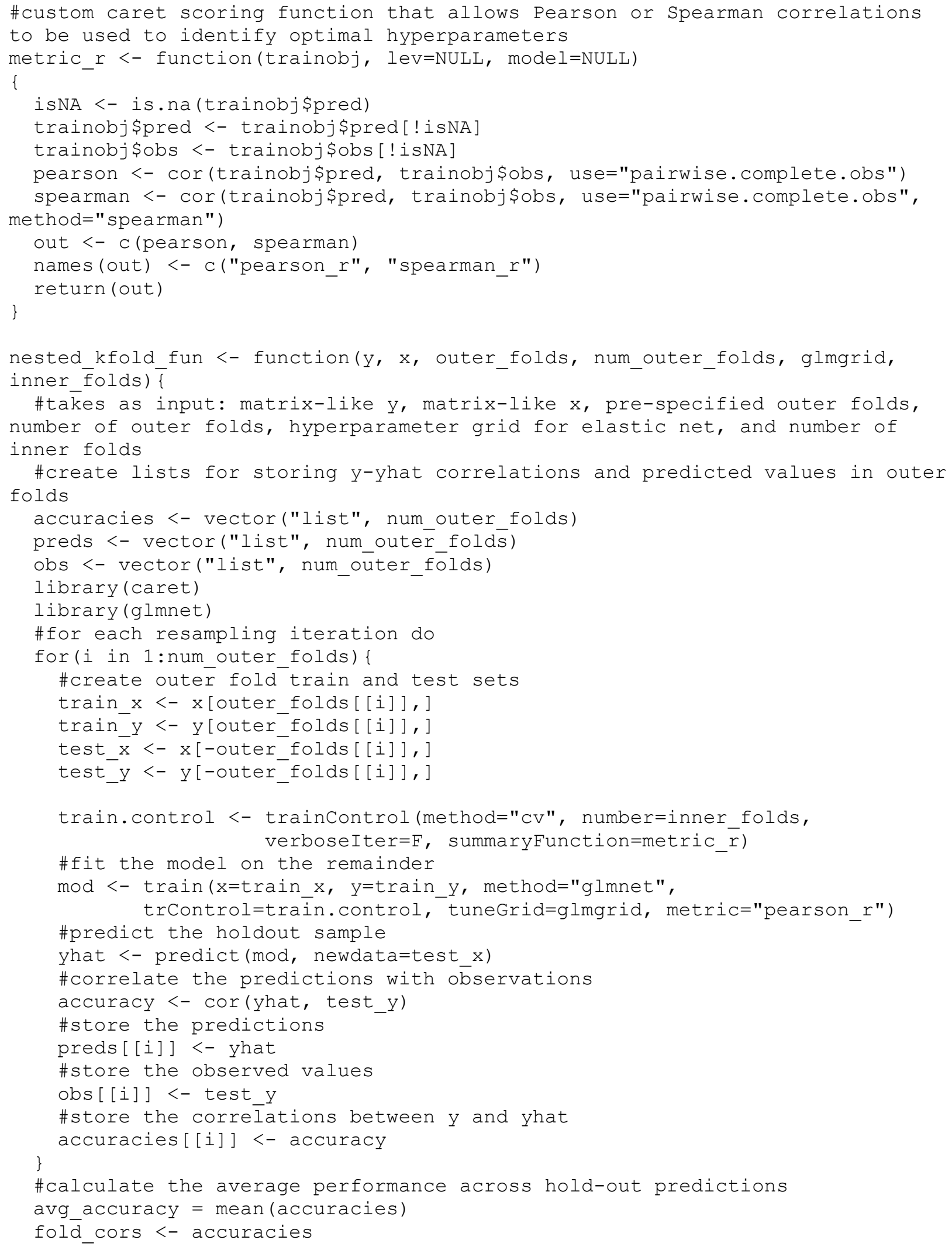




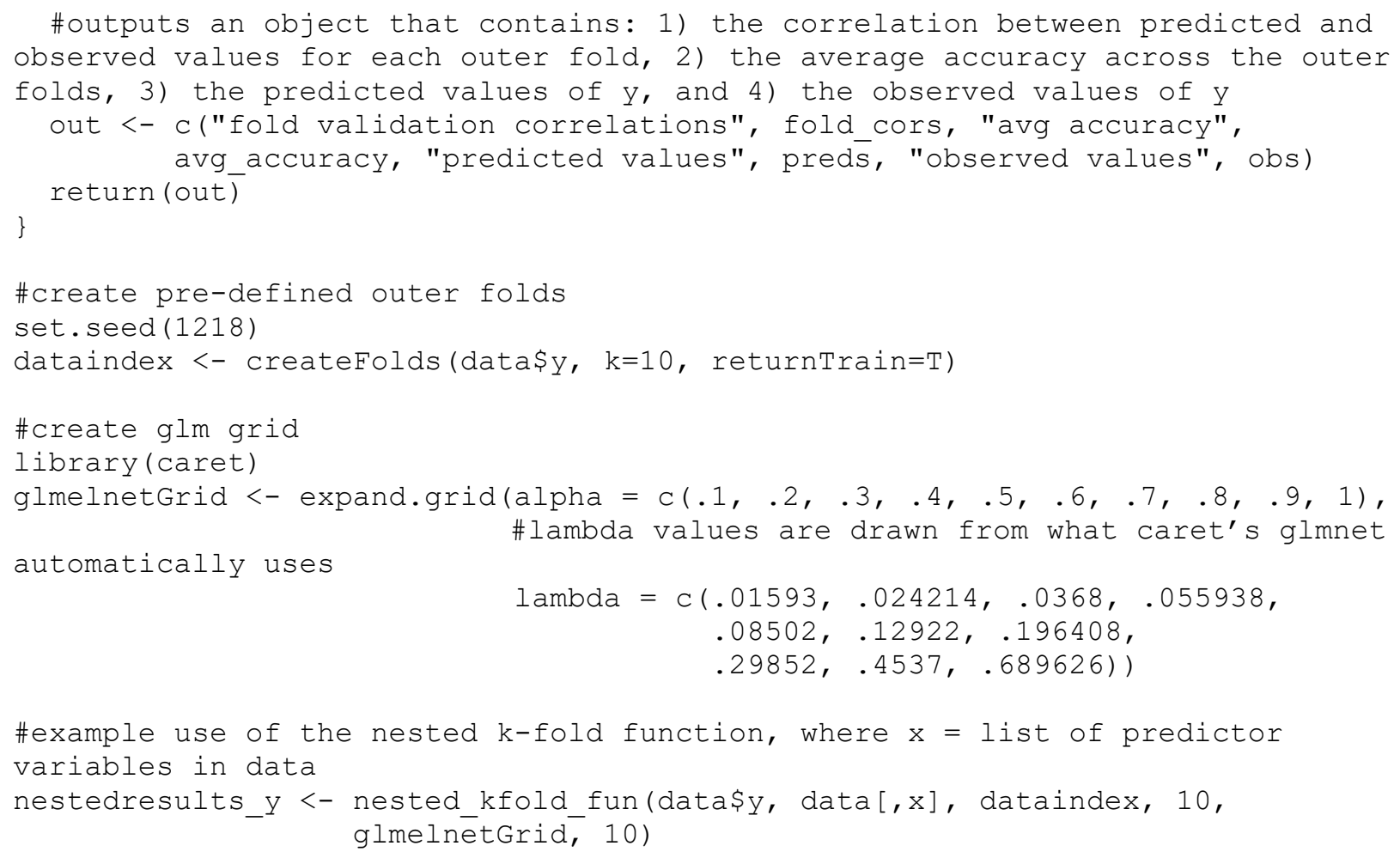

